\title{
21
}

\section{Relaxation Projections and the Method of Moments}

\author{
Ulf Dieckmann and Richard Law
}

\subsection{Introduction}

Theory in spatial ecology has to steer a narrow and challenging course between the Scylla of oversimplification and the Charybdis of intractability. Until about 15 years ago, most of theoretical ecology was based on the mean-field paradigm, thus targeting well-mixed ecological systems. Although the underlying assumption of spatial homogeneity is violated for many, if not most, ecological populations and communities in the field, mean-field approaches appeared to be the only way forward. They even took center stage in certain areas, as in epidemiological systems (Bailey 1975; Anderson and May 1991), today recognized as typical examples of ecological processes for which space matters. It was only with the advent and ready availability of modern computer technology that explorations into critical effects of spatial heterogeneities became feasible (Levin 1974, 1976; Weiner and Conte 1981; Weiner 1982; Pacala and Silander 1985; Holsinger and Roughgarden 1985; Pacala 1986; Hogeweg 1988). Today, computer screens and journals abound with images of spatially extended simulations that have convincingly demonstrated that many predictions of classical ecological theory are inappropriate in the presence of spatially structured habitats or short-range ecological interactions.

Despite their value as counterexamples to mean-field predictions and their usefulness in exploring the emergence of macroscopic effects resulting from microscopic ecological mechanisms, simulation studies often remain inconclusive. Are the reported phenomena robust under changed ecological parameters? Where, among the noisy dynamics of individual-based and stochastic models, is the ecological signal? How many (usually timeconsuming) spatial simulations have to be run before reliable conclusions can be drawn? These questions remind us that only part of our ecological 
understanding is based on description: on top of this, we look for mechanistic explanations and for reliable generalizations from observations. Specifically, we are interested in qualitative rules, and for this reason we would like heterogeneous ecological processes to be amenable to tools that allow robust conclusions to be drawn. For many systems, such sound qualitative insight can only be derived from careful quantitative analyses (well-known examples from classical ecological theory are many inequality conditions and results of bifurcation analyses).

Is it realistic to hope for a middle ground between oversimplified meanfield models and intractable computer simulations? The answer depends on how many essential degrees of freedom there are in spatial ecological systems. A degree of freedom here is a quantitative piece of information needed to specify the current state of and the expected change in a given system. How many degrees of freedom are considered essential often depends on the purpose of an investigation. Think of the trajectory traced by a stone thrown into the air. A detailed description of the flying stone would account for the state of all its atoms. For practical predictions of the stone's expected path, however, most of these details are utterly irrelevant: only the position and velocity of its center of mass are essential, and even the stone's orientation and rotational speed can be neglected. Another illustration is provided by milk being poured into a cup of coffee. To specify the initially intricate pattern of milk and coffee mixing, very many variables are needed. After a short while, however, the milk concentration becomes uniform and can be specified by a single number. Likewise, in ecological systems we can often ignore most of the physiological or biochemical details of individuals, provided that our interest rests at the population level. Lewontin has introduced the term dynamic sufficiency for distinguishing between essential and dispensable degrees of freedom: a subset of variables is called dynamically sufficient if sufficiently accurate predictions of future dynamics can be based on these variables alone (Lewontin 1974).

Sometimes the dynamically sufficient number of variables is small from the outset (the thrown stone), and sometimes it quickly decreases as a consequence of internal processes (the milk drop). The rapid "destruction" of degrees of freedom is also typical for many spatially extended systems: often, a small set of variables is dynamically sufficient for adequately capturing the system's state. In a grassland, for instance, observing a few spatial statistics in a few square meters may provide most of the information required to characterize the whole area. We do not need to know the precise position of every single shoot in this area to predict the system's expected 
Box 21.1 Relaxation projections in spatial ecology

Spatio-temporal processes usually possess some degrees of freedom operating on a fast time scale: any initial pattern $p$, taken from the set $P$ of all possible patterns, will quickly converge to a more limited set $P_{r}$ of patterns. A smaller number of variables - called essential degrees of freedom - is required to characterize patterns within $P_{r}$ than within $P$.

Spatial statistics are variables for describing the state of a spatiotemporal process at any point in time. If $S$ denotes a set of spatial statistics sufficient to characterize patterns in $P$, and $S_{r}$ contains those needed for $P_{r}$, the statistics in $S^{\prime}=S \backslash S_{r}$ are only needed to distinguish between patterns in $P^{\prime}=P \backslash P_{r}$ and hence are no longer essential after the system's fast degrees of freedom have decayed.

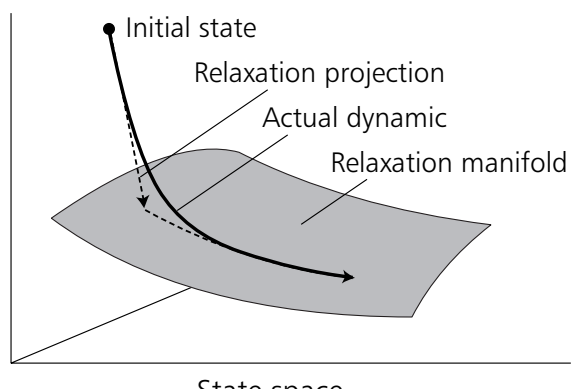

State space

Illustration of the rapid decay of initial conditions toward a relaxation manifold in a threedimensional dynamic. The relaxation projection defines a relaxation manifold and a projection of initial conditions onto this manifold; both are supposed to approximate the actual dynamics' first phase of rapid decay.

As $S^{\prime}$ thus retains no essential information about the state of the process after relaxation, all spatial statistics of resulting patterns can be expressed approximately as functions of those in $S_{r}, S^{\prime}=f\left(S_{r}\right)$. Hence, the functions $f$ simplify the complexity of describing the state of a spatial process. In addition, the functions $f$ simplify the dynamics of the process: in the space $S$ of statistics, the functions $f$ define

a projection of arbitrary initial conditions $s=\left(s_{r}, s^{\prime}\right)$ in $S$ onto a relaxation manifold, $\left(s_{r}, s^{\prime}\right) \rightarrow\left(s_{r}, f\left(s_{r}\right)\right)$, and

the shape of the relaxation manifold itself, which is the subspace of all states $s$ in $S$ invariant under that projection, thus satisfying $\left(s_{r}, s^{\prime}\right)=$ $\left(s_{r}, f\left(s_{r}\right)\right)$.

The projection mimics the fast relaxation of the spatio-temporal process. The relaxation manifold can be used for constructing a simplified dynamic on that manifold: starting from a state $s_{r}$ on the relaxation manifold, the missing statistics $s^{\prime}$ can be reconstructed as $s^{\prime}=f\left(s_{r}\right)$. Now the change $\frac{d}{d t} s$ of the state $s=\left(s_{r}, s^{\prime}\right)$ follows from the dynamics of the spatiotemporal process and implies a change $\frac{d}{d t} s_{r}$ along the relaxation manifold. 


\section{Box 21.1 continued}

We call projections of this kind relaxation projections. In general, projections are non-invertible mappings, and objects projected consequently carry a diminished amount of information. In particular, relaxation projections remove the dynamically nonessential information from a spatial pattern. The art of constructing relaxation projections amounts to finding suitable small sets of statistics $S$ and simple functions $f$ so that the actual relaxation process as well as the resulting relaxation manifold are well approximated and the relaxation projection itself is sufficiently simple to allow for the derivation of tractable dynamics.

development at the population level: specifying a much smaller number of essential degrees of freedom will suffice. But only in systems that eventually become completely homogeneous, like the milk in the cup of coffee, does the number of essential degrees of freedom become minimal. In other cases, a certain amount of heterogeneity is preserved in the system since certain mechanisms counteract full mixing. In ecological systems, these mechanisms arise from the local interactions between individuals, their restricted dispersal, and their dependence on a potentially heterogeneous habitat; we therefore cannot simply expect all variables apart from mean densities to become superfluous. Instead, a balance between forces of mixing and ordering (Watt 1947) will lead to states with more essential degrees of freedom than in mean-field approximations, but still with only a fraction of the vast number of degrees of freedom possible.

This general expectation is corroborated by observations and explicit simulations of spatial ecological dynamics that start from arbitrarily complicated initial configurations and whose internal dynamics, after a while, reliably sustain only a small class of patterns. Imagine gardeners setting up a plot with ornate patterns of plants, like those in the baroque gardens at Versailles. Without continuous examination and lots of effort on the part of the gardeners, that intricate distribution will soon relax into a more natural configuration, characterized by considerably fewer essential degrees of freedom than were initially present. Interactions between plants tend to operate at a local scale; therefore, in the absence of external heterogeneities, any long-range correlations between plants present in the gardeners' original setup cannot persist - leaving the Versailles gardens unattended for a century would eradicate most of the artful patterns originally devised. In our quest for a tractable but still dynamically sufficient description of ecological systems in space and time, we can capitalize on this tendency 
Box 21.2 The Dirac delta function in spatial ecology

Dirac's delta function is a particularly useful tool for characterizing the distribution of individuals across continuous spaces. Imagine a plot of plants, each occupying a specific location. One way of describing their spatial distribution relies on quadrat counts: after imposing a square grid on the ecological space, the number of plant individuals within each square is counted. The resulting information can be represented as a histogram, where the volume of each column is proportional to the number of individuals present in the underlying square (Figure a).

(a)

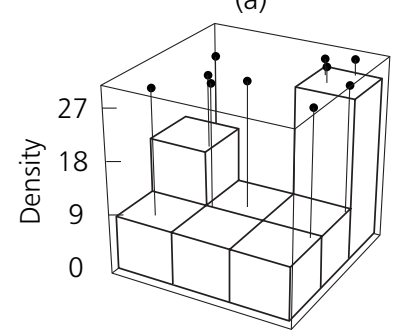

(b)

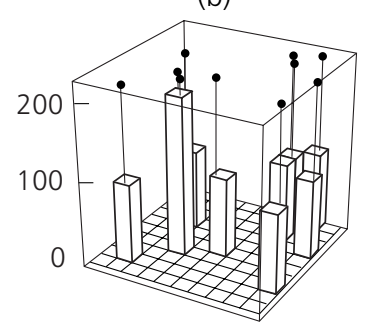

(c)

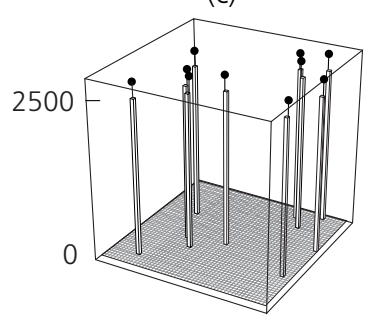

Histograms of increasing resolution, constructed for the same spatial pattern of individuals distributed over the unit square (depicted as points on the top plane). The high-resolution limit yields a Dirac delta function at the location of each individual.

The information captured by quadrat counts, however, is incomplete. Within each square of the grid, no information is available about the distribution of individuals. The situation can be improved by choosing a grid of higher resolution in which each square is of a smaller size. For a sufficiently fine grid, most of the squares will contain either zero or one individual, with squares containing two or more individuals being the exception. If the volume of histogram columns is still to correspond to the number of individuals, the height of columns has to be increased as their base squares are shrunk (Figure b). To assess the number of individuals within a certain area, we can then just add the volume of all histogram columns over that area.

Plants and animals, of course, possess a spatial extension as well as a spatial location. It is often convenient to distinguish these two aspects of spatial structure by thinking of individuals as being centered at some location, while including information about their extension, if necessary, in the state of individuals. Although on finer grids accuracy is improved, information regarding the precise location of individuals within squares is still discarded.

For theoretical considerations, then, it is natural to let the size of squares shrink to zero, so that full information about the location of individuals is retained. Keeping our interpretation of the volumes of histogram columns requires letting their height go to infinity. From this limit we obtain a Dirac 


\section{Box 21.2 continued}

delta function at the location of each individual. These functions are zero everywhere, except at one specific location. There, their height is infinite, such that their integral is 1 (for illustration of this point, a very fine grid is shown in Figure c). By integrating over all delta functions within a given area, we thus obtain the abundance of individuals occupying that area.

In summary, a spatial pattern of individuals can be described by a sum of delta functions, each peaked at the location of a single individual, thus describing its contribution to the population's density function.

of natural systems to "destroy" degrees of freedom by relaxation. Hence, for models of ecological heterogeneity, we have to cast the set of all possible configurations onto that much smaller subset toward which the system will rapidly relax; we call this general procedure a relaxation projection (see Box 21.1). Relaxation projections help establish the middle ground between oversimplification and intractability. By culling the dynamically irrelevant degrees of freedom initially present in many spatially heterogeneous systems, we reduce the number of variables to manageable proportions. Obviously, this culling must not go too far: retaining only mean densities would take us back to mean-field descriptions.

These considerations lead to the following question: if we are to capture the essential aspects of spatial heterogeneity in a dynamically sufficient way, which variables should we use to complement mean densities? To address that central issue in detail, this chapter uses a widely applicable class of stochastic models for individual-based and spatially extended ecological systems, described in Section 21.2. In Section 21.3, we introduce and analyze a flexible set of spatial statistics, called correlation densities or spatial moments, which are candidates for providing the extra spatial information needed on top of mean densities. Section 21.4 explains how relaxation projections can be applied to the dynamics of spatial moments. We examine alternative projection schemes and show that some of these provide powerful descriptions of spatially heterogeneous ecological change. Section 21.5 considers extensions of the framework and examines consequences of the stochastic effects that inevitably arise when individuals interact with finite numbers of neighbors. We conclude that the novel class of ecological models developed in Sections 21.3 and 21.4 allows for robust and generalizable insights into the inner workings of spatially structured ecological populations and communities. 


\subsection{Individual-based Dynamics in Continuous Space}

In this section we introduce a class of spatially explicit, individual-based, stochastic birth-death-movement processes that, on the one hand, can be calibrated to reflect ecological dynamics as observed in the field and, on the other hand, are suitable targets for applying the technique of relaxation projections (Sections 21.3 and 21.4). Such individual-based stochastic processes in continuous space also underlie the analyses in Chapter 20, and it is helpful to make them explicit.

\section{Patterns of individuals in continuous space}

A natural starting point for specifying ecological details of a spatially explicit model is the demographic events experienced by individuals. As a step toward the real world, we allow birth and death rates of individuals to depend on their local environment. In the class of models below, individuals can disperse (at birth) and relocate (during their lifetime) within a given habitat. We think it is preferable to envisage individuals at locations in continuous space rather than at discrete sites on a grid. Avoiding the discretization of space into regular cells - which, for most ecological systems, is artificial - offers a more faithful and direct correspondence between model parameters and model dynamics and those quantities and processes that can be measured in the field. This modeling framework has the additional advantage of adequately reflecting the gradual effects of increased physical distance on the interaction strength between individuals, and it allows for continuous changes of dispersal and relocation probabilities with distance traveled.

Individuals can belong to different species $i$, with $i=1, \ldots, n$, where $n$ is the number of species in the community. All individuals inhabit a space of locations $x$; the habitat can have one, two, or three spatial dimensions. Most applications in plant ecology, of course, focus on planar habitats. The spatial extension of the habitat is measured by $A$, denoting the habitat's length, area, or volume, respectively. Individuals $l$ in species $i$ are situated at spatial locations $x_{i l}$, with $l=1, \ldots, A N_{i}$, where $N_{i}$ is the mean population density of species $i$. The distribution of individuals in each species $i$ is described by spatial density functions $p_{i}(x)=\sum_{l} \delta_{x_{i l}}(x)$, where $\delta_{x_{i l}}$ denotes Dirac's delta function peaked at location $x_{i l}$ (for an explanation of this representation and the motivation behind it, see Boxes 21.2 and 21.3). The density function $p_{i}(x)$ is thus peaked at all locations occupied by individuals of species $i$ and is zero elsewhere. At any moment in time, the spatial pattern within the community is given by collecting the density functions of all species into a vector $p(x)=\left(p_{1}(x), \ldots, p_{n}(x)\right)$. 
Box 21.3 Properties of the Dirac delta function

The delta function was introduced by the English physicist Paul A.M. Dirac (Dirac 1926, 1958), and relates to previous work by G. Kirchhoff and O. Heaviside (Jammer 1966). Dirac's idea was to construct a strictly localized function on the real numbers: $\delta(x)$ is zero for any $x$, except for $x=0$, where it is peaked.

To make this notion precise, the delta function is defined by

$$
\int_{-\infty}^{+\infty} f\left(x^{\prime}\right) \delta\left(x^{\prime}-x\right) d x^{\prime}=f(x)
$$

for any continuous function $f$. This is called the sifting property of the delta function: since the term $\delta\left(x^{\prime}-x\right)$ is 0 except at $x^{\prime}=x$ (where the delta function's argument vanishes), only at that point can the value of $f$ contribute to the integral. This can also be expressed by $f\left(x^{\prime}\right) \delta\left(x^{\prime}-x\right)=$ $f(x) \delta\left(x^{\prime}-x\right)$.

From this definition we can derive other useful properties of the delta function:

- The delta function is symmetric: $\delta(-x)=\delta(x)$.

Its integral equals 1: $\int_{-\infty}^{+\infty} \delta\left(x^{\prime}\right) d x^{\prime}=1$.

- One or more zeros in the delta function's argument contribute according to their inverse slope: $\delta(g(x))=\sum_{i} \delta\left(x-x_{i}\right) /\left|g^{\prime}\left(x_{i}\right)\right|$, summing over all zeros $x_{i}$ of $g$.

- Its primitive function is the unit-step or Heaviside function: $\int_{-\infty}^{x} \delta\left(x^{\prime}\right) d x^{\prime}=\theta(x)$, where $\theta(x)$ equals 0 for negative and 1 for positive $x$.

- The Fourier and Laplace transforms of the delta function are equal to 1.

The Dirac delta function can also be envisaged as the limit of a series of functions. Setting $h(x)$ to $1 / \varepsilon$ for $|x|<\varepsilon / 2$ and to 0 elsewhere, we can write $\delta(x)=\lim _{\varepsilon \rightarrow 0} h(x)$. Compare this with the figures in Box 21.2: while the width of such a function shrinks to zero, its height goes to infinity. Setting $h$ to a normal distribution with mean 0 and standard deviation $\varepsilon$ has the same effect. All properties of the delta function can alternatively be derived from such limit representations (theory of distributions; Schwartz 1950).

In more than one dimension, the delta function is defined as the product of one-dimensional delta functions: $\delta(x)=\delta\left(x_{1}\right) \delta\left(x_{2}\right) \ldots \delta\left(x_{n}\right)$ for $x=$ $\left(x_{1}, x_{2}, \ldots, x_{n}\right)$. For convenience, the location of a delta function's peak is often given as a subscript: $\delta\left(x^{\prime}-x\right)=\delta_{x}\left(x^{\prime}\right)$. 


\section{Birth, death, and movement events}

The per capita death rates of individuals can depend on the presence or absence of other individuals in their local environment. We denote by $w_{i j}^{(d)}\left(x-x^{\prime}\right)$ the strength with which an individual of species $j$ at location $x^{\prime}$ affects the mortality of an individual of species $i$ at location $x$. The functions $w_{i j}^{(d)}$ are called interaction kernels for density-dependent death and are scaled so that they integrate to $1, \int w_{i j}^{(d)}(\xi) d \xi=1$. (In this chapter we denote absolute spatial locations by $x \mathrm{~s}$ and relative locations by $\xi \mathrm{s}$.) The reason for calling these functions kernels becomes evident when we realize that, in the death rate of an individual of species $i$ at location $x$ in a community with spatial pattern $p$,

$D_{i}(x, p)=d_{i}+\sum_{j} d_{i j}^{\prime} \int w_{i j}^{(d)}\left(x^{\prime}-x\right)\left[p_{j}\left(x^{\prime}\right)-\delta_{i j} \delta_{x}\left(x^{\prime}\right)\right] d x^{\prime}$,

$w_{i j}^{(d)}$ occurs as the kernel of a convolution integral (see also Boxes 20.1 and 20.2). Here, $d_{i}$ denotes the intrinsic per capita death rate of species $i$, and $d_{i j}^{\prime}$ weighs the density-dependent effect of species $j$ on the mortality of individuals in species $i$. The integral in Equation (21.1a) collects the contributions from all locations $x^{\prime}$ according to their interaction strength $w_{i j}^{(d)}\left(x^{\prime}-x\right)$ and local density $p_{j}\left(x^{\prime}\right)$. Obviously, individuals do not compete with themselves; therefore the contribution of the focal individual at location $x$, given by $\delta_{x}\left(x^{\prime}\right)$, is removed from the integration when the summation over $j$ reaches the focal species $i$ (the Kronecker symbol $\delta_{i j}$ equals 1 for $i=j$ and is 0 otherwise).

The rate of movement of an individual in species $i$ at location $x$ to another location $x^{\prime}$ is given by

$$
M_{i}\left(x, x^{\prime}, p\right)=m_{i}\left(x^{\prime}-x\right) .
$$

Equation (21.1b) implies that rates of movement events are homogeneous in space; they only depend on the distance moved $x^{\prime}-x$, and not on absolute location $x$ or on spatial pattern $p$. Nevertheless, the movement kernel $m_{i}$ can differ from species to species. It is convenient to keep the movement kernel unnormalized: its integral $\left|m_{i}\right|=\int m_{i}(\xi) d \xi$ measures the expected total per capita rate of movement.

Like death events, we allow the per capita reproduction rate for each individual to depend on its local environment. Unlike death events, however, an offspring takes, by means of dispersal, a spatial location different from that of its parent. The rate of reproduction of an individual in species $i$ at 
location $x$, giving rise to a new individual at location $x^{\prime}$, is given by

$$
\begin{aligned}
B_{i}\left(x, x^{\prime}, p\right) & =\left[b_{i}+\sum_{j} b_{i j}^{\prime} \int w_{i j}^{(b)}\left(x^{\prime \prime}-x\right)\right. \\
& \left.\times\left[p_{j}\left(x^{\prime \prime}\right)-\delta_{i j} \delta_{x}\left(x^{\prime \prime}\right)\right] d x^{\prime \prime}\right] m_{i}^{(b)}\left(x^{\prime}-x\right) .
\end{aligned}
$$

Here $b_{i}$ and $b_{i j}^{\prime}$ denote the density-independent and density-dependent components of the per capita birth rate, respectively; $w_{i j}^{(b)}$ is the interaction kernel for density-dependent birth and $m_{i}^{(b)}$ is the dispersal kernel. Like the kernels of interaction, the dispersal kernel is normalized to 1. In Equation (21.1c), competitive interactions occur for $b_{i j}^{\prime}<0$; these will be the most natural choice for many ecological systems. Yet, mixtures of competition with neutral $\left(b_{i j}^{\prime}=0\right)$ or mutualistic $\left(b_{i j}^{\prime}>0\right)$ interactions are also possible and can be readily incorporated. Equations (21.1a) and (21.1c) describe the spatial analogues of Lotka-Volterra competition and provide a natural starting point for exploring interaction effects in spatially heterogeneous systems. Alternative assumptions, allowing for nonlinear dependencies of $D_{i}$ and $B_{i}$ on $p$, are discussed in Section 21.5.

\section{Pattern dynamics}

Starting from an initial spatial configuration, we can now investigate how a pattern changes with the ecological events described above. According to Equations (21.1), the dynamics of patterns are stochastic (any two runs from the same starting patterns are expected to result in different patterns) and Markovian (rates of change at any moment in time depend only on the current pattern). Such processes are characterized by so-called master equations (van Kampen 1992). Denoting by $P(p)$ the probability density for observing pattern $p$, the rate of change in this probability density is given by

$$
\frac{d}{d t} P(p)=\int\left[w\left(p \mid p^{\prime}\right) P\left(p^{\prime}\right)-w\left(p^{\prime} \mid p\right) P(p)\right] d p^{\prime}
$$

where $w\left(p^{\prime} \mid p\right)$ is the probability density per unit time that any event will turn a pattern $p$ into another pattern $p^{\prime}$. Hence the first term on the righthand side corresponds to an increase in the probability density of pattern $p$ due to events that lead to $p$ and originate from a different pattern $p^{\prime}$, while the second term captures the decrease of the probability density at $p$ resulting from events that change pattern $p$ into a different pattern $p^{\prime}$. 
Box 21.4 The generalized delta function

The generalized delta function $\Delta$ extends the functionality of the Kronecker symbol and the Dirac delta function from the realm of integers and real numbers, respectively, to that of functions.

Markovian jump processes on integers, reals, or functions are described by master equations (for an introduction, see, e.g., van Kampen 1992). This class of equations is based on transition rates and characterizes the flow of probability into and out of states of a stochastic process, as in Equation (21.2a). Transitions between states often result from different types of event, each of which changes the state of the process in a particular manner. Switch functions are used to link descriptions of events and their rates to transition rates of the process; these functions are "on" only for a particular transition. An important role for all three delta functions is to serve as such switches.

\begin{tabular}{ll}
\hline State variable & Master equation/ transition rates / switch function \\
\hline Integer $i$ & $\frac{d}{d t} P_{i}=\sum_{i^{\prime}}\left[w_{i i^{\prime}} P_{i^{\prime}}-w_{i^{\prime} i} P_{i}\right]$ \\
& $w_{i^{\prime} i}=\sum_{\Delta i} E_{\Delta i, i} \delta_{i+\Delta i, i^{\prime}}$ \\
& $\sum_{i^{\prime}} F_{i^{\prime}} \delta_{i^{\prime} i}=F_{i}$ \\
& $\frac{d}{d t} P(r)=\int\left[w\left(r \mid r^{\prime}\right) P\left(r^{\prime}\right)-w\left(r^{\prime} \mid r\right) P(r)\right] d r^{\prime}$ \\
Real number $r$ & $w\left(r^{\prime} \mid r\right)=\sum_{\Delta r} E(\Delta r, r) \delta\left(r+\Delta r-r^{\prime}\right)$ \\
& $\int F\left(r^{\prime}\right) \delta\left(r^{\prime}-r\right) d r^{\prime}=F(r) \quad($ Dirac delta function) \\
& $\frac{d}{d t} P(f)=\int\left[w\left(f \mid f^{\prime}\right) P\left(f^{\prime}\right)-w\left(f^{\prime} \mid f\right) P(f)\right] d f^{\prime}$ \\
& $w\left(f^{\prime} \mid f\right)=\sum_{\Delta f} E(\Delta f, f) \Delta\left(f+\Delta f-f^{\prime}\right)$ \\
& $\int F\left(f^{\prime}\right) \Delta\left(f^{\prime}-f\right) d f^{\prime}=F(f)($ Generalized delta function) \\
\hline
\end{tabular}

While the sums in the equations for transition rates $w$ extend over all possible jumps $\Delta i, \Delta r$, or $\Delta f$, only permitted jumps with non-vanishing event rates $E$ contribute to the transition rates $w$. Substituting the transition rates into the corresponding master equation, the switch functions select only those states $i^{\prime}, r^{\prime}$, or $f^{\prime}$ that can be reached by permitted jumps.

We can envisage the generalized delta function $\Delta$ as an infinitely narrow and infinitely high peak in the space of functions $f$. Such a heuristic notion, together with the functional integration occurring in the master equation for functions, is made exact by defining the generalized delta function so as to collapse the functional integration in $\int F\left(f^{\prime}\right) \Delta\left(f^{\prime}-f\right) d f^{\prime}$ by yielding the functional $F$ 's value at the location of $\Delta$ 's peak, $F(f)$. This definition is analogous to that of Dirac's continued 


\section{Box 21.4 continued}

delta function (Box 21.3). After the functional transition rates are thus combined with the functional master equation, neither a functional integration nor a generalized delta function remain in the end result. Or, in the words of Dirac (1958): "The use of delta functions thus does not involve any lack of rigour in the theory, but is merely a convenient notation, enabling us to express in a concise form certain relations which we could, if necessary, rewrite in a form not involving delta functions, but only in a cumbersome way which would tend to obscure the argument."

The functions $w\left(p^{\prime} \mid p\right)$ are also known as transition rates and for our model are simply given by summing and integrating over all possible birth, death, and movement events that can turn a pattern $p$ into a pattern $p^{\prime}$,

$$
\begin{aligned}
w\left(p^{\prime} \mid p\right) & =\sum_{i} \iint B_{i}\left(x, x^{\prime}, p\right) p_{i}(x) \Delta\left(p+u_{i} \delta_{x^{\prime}}-p^{\prime}\right) d x d x^{\prime} \\
& +\sum_{i} \int D_{i}(x, p) p_{i}(x) \Delta\left(p-u_{i} \delta_{x}-p^{\prime}\right) d x \\
& +\sum_{i} \iint M_{i}\left(x, x^{\prime}, p\right) p_{i}(x) \Delta\left(p-u_{i} \delta_{x}+u_{i} \delta_{x^{\prime}}-p^{\prime}\right) d x d x^{\prime} .
\end{aligned}
$$

The unit vector $u_{i}$ for species $i$ has 1 as its $i$ th element and zeros elsewhere. We refer to $\Delta$ as the generalized delta function (Dieckmann 1994; Dieckmann et al. 1997, in press), see Box 21.4. Like Dirac's delta function $\delta$ (Boxes 21.2 and 21.3), it is peaked where its argument is zero, and is zero elsewhere. Unlike Dirac's delta function, however, generalized delta functions take functions as arguments. All three sums on the right-hand side of Equation (21.2b) comprise terms of the form $\Delta\left(p_{\text {event }}-p^{\prime}\right)$. They therefore only contribute to the transition rate $w\left(p^{\prime} \mid p\right)$ when $p_{\text {event }}=p^{\prime}$, in other words, only if the considered event can turn the current pattern $p$ into the new pattern $p^{\prime}$. For example, the expression $\Delta\left(p+u_{i} \delta_{x^{\prime}}-p^{\prime}\right)$ is "switched on" when $p+u_{i} \delta_{x^{\prime}}=p^{\prime}$ (that is, only if a birth event in species $i$, producing a new individual at location $x^{\prime}$, changes pattern $p$ into pattern $p^{\prime}$ ).

Models of the kind described in this section are flexible tools for studying the dynamics of spatially extended ecological systems. Their parameterization gets closer than mean-field models to individual-based processes in the field and should permit incorporation of empirical measurements. Moreover, their intrinsic mixture of randomness and determinism 
(a)
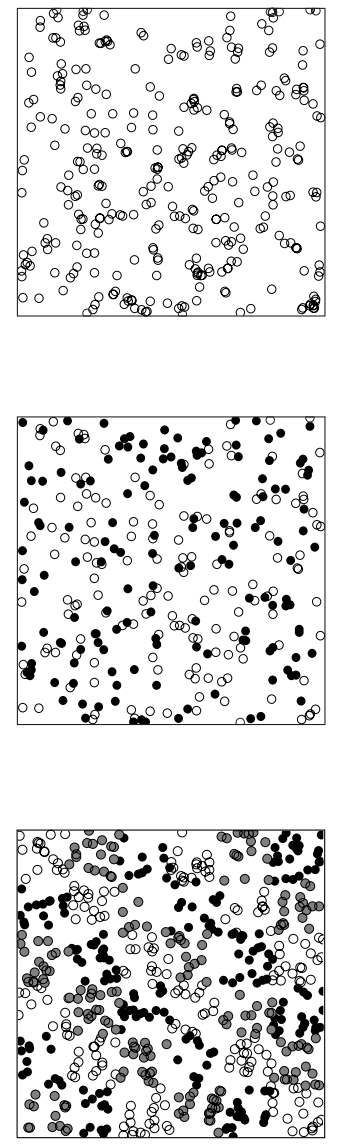

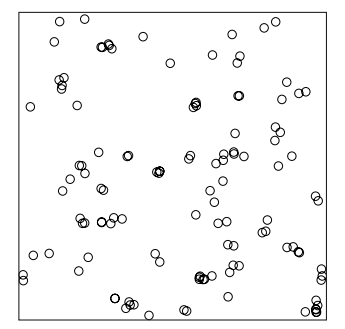

(b)
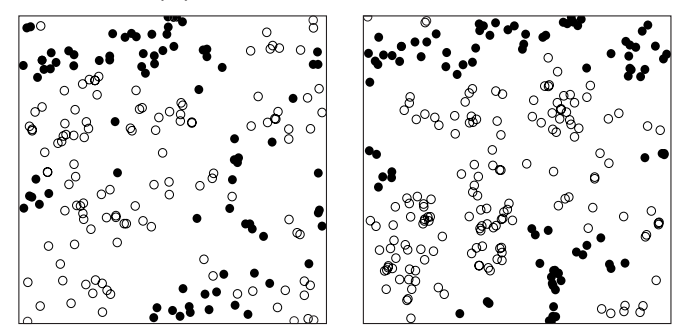

(c)

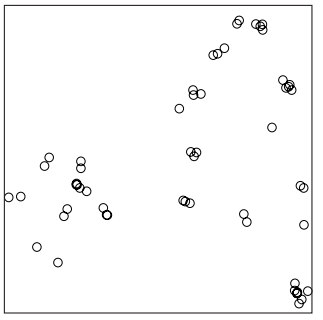

8.
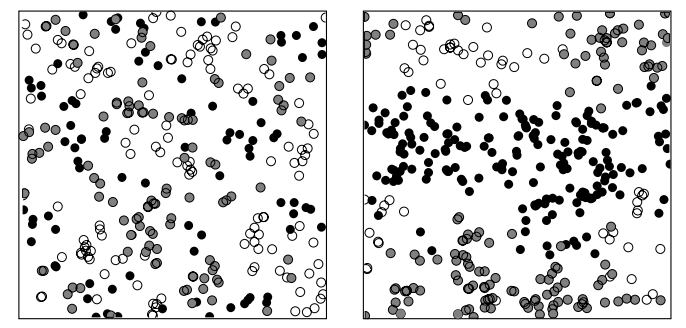

Figure 21.1 Examples of individual-based birth-death-movement processes. (a) A single species with dispersal and local logistic competition. Owing to spatial aggregation, the asymptotic mean density is significantly below the mean-field prediction. (b) Two dispersing species with competitive interactions. Under mean-field conditions, this pair of species cannot coexist. (c) Three dispersing species with competitive interactions. Notice how quickly any regularity of the initial pattern is destroyed. For all three systems, stochastic realizations are shown at three different moments in time: initial pattern (left column), intermediate pattern (center column), and a pattern from the asymptotically stable distribution (right column). Parameters for all three systems are given in Table 21.1.

corresponds well to processes in natural populations. Models of this kind are also easily implemented as computer simulations (see Figures 21.1, 14.1, and 14.5). Thus individual realizations of the stochastic processes defined above are readily available; their properties can be investigated in detail without introducing any further simplifying assumptions. At the same time, these complicated interactive dynamics are ideal candidates for demonstrating how to simplify spatial complexity. As we will see in the following two sections, considerable insight can be gained by subjecting these dynamics to relaxation projections and to the resulting method of moments. 
Table 21.1 Parameter values for the three systems in Figure 21.1.

\begin{tabular}{lll}
\hline System & Times $^{a}$ & Parameters $^{b}$ \\
\hline Figure 21.1a & $0,10,30$ & $N_{1}(0)=200$ \\
& & $b_{1}=0.4, d_{1}=0.2, d_{11}^{\prime}=0.001$ \\
& s.d. $w_{11}^{(d)}=0.04$, s.d. $m_{1}^{(b)}=0.02$ \\
& & $N_{1}(0)=N_{2}(0)=150$ \\
& $b_{1}=b_{2}=0.6, d_{1}=d_{2}=0.2$ \\
& $d_{11}^{\prime}=d_{22}^{\prime}=0.001, d_{12}^{\prime}=0.004, d_{21}^{\prime}=0.005$ \\
& s.d. $w_{11}^{(d)}=$ s.d. $w_{12}^{(d)}=$ s.d. $m_{1}^{(b)}=$ s.d. $m_{2}^{(b)}=0.06$ \\
& s.d. $w_{21}^{(d)}=$ s.d. $w_{22}^{(d)}=0.05$ \\
& $N_{1}(0)=N_{2}(0)=N_{3}(0)=200$ \\
& & $b_{1}=b_{2}=b_{3}=0.8, d_{1}=d_{2}=d_{3}=0.2$ \\
& $d_{11}^{\prime}=d_{22}^{\prime}=d_{33}^{\prime}=0.001, d_{12}^{\prime}=0.003, d_{13}^{\prime}=0.004$ \\
& $d_{21}^{\prime}=0.005, d_{23}^{\prime}=0.003, d_{31}^{\prime}=0.002, d_{32}^{\prime}=0.006$ \\
& s.d. $w_{11}^{(d)}=$ s.d. $w_{12}^{(d)}=$ s.d. $w_{13}^{(d)}=$ s.d. $w_{21}^{(d)}=$ \\
& s.d. $w_{22}^{(d)}=$ s.d. $w_{23}^{(d)}=$ s.d. $w_{31}^{(d)}=$ s.d. $w_{32}^{(d)}=$ \\
& s.d. $w_{33}^{(d)}=0.06$ \\
& s.d. $m_{1}^{(b)}=0.06$, s.d. $m_{2}^{(b)}=0.07$, s.d. $m_{3}^{(b)}=0.05$ \\
\hline
\end{tabular}

$a^{a}$ Times at which the three snapshots are taken. Each snapshot depicts the unit square with periodic boundary conditions.

${ }^{b}$ Interaction and dispersal kernels are Gaussian with standard deviations (s.d.) as indicated. Parameters not mentioned are zero.

\subsection{Dynamics of Correlation Densities}

With the general class of birth-death-movement models in place (Section 21.2), we consider how to simplify the spatial complexity of these models by applying a relaxation projection. As a first step in this process, we have to decide which are the essential degrees of freedom in the spatial patterns under consideration.

\section{Scales of spatial heterogeneity}

Spatial heterogeneity can occur at various scales, and therefore a basic distinction is helpful here:

- either patterns are small scale relative to the extension of the habitat, in which case the whole habitat contains many varied instances of a unit pattern,

- or we are dealing with large-scale patterns, for which the habitat is not large enough to comprise many replicates of any unit pattern.

The term "unit pattern" here is not meant in the sense of a spatial tiling, but rather refers to the totality of spatial configurations at the scale above 
which the pattern becomes repetitive and conveys no further information on essential degrees of freedom (for a way of estimating this scale, see Chapter 12). Think, for example, of an ecological habitat that is divided into two disjunct spatial domains, each of which is occupied by a single species. Here, the pattern of spatial segregation spans the entire habitat and thus occurs at a large scale relative to this habitat. In contrast, imagine that local clumps are formed by individuals of one species, and that these are gradually invaded by individuals of a second species, while at the same time new clumps of the former are re-established elsewhere. In this case, a not-too-small habitat will comprise many clumps and we therefore refer to the pattern as being small scale (with the various shapes of clumps playing the role of "unit patterns"). The dichotomy is important because essential degrees of freedom for large-scale patterns vary from pattern to pattern. This means that for each type of large-scale pattern, it would be necessary to evaluate which essential degrees of freedom best capture the system's state. For small-scale patterns, however, matters are simpler: here, average information on the local environments of individuals often can capture the essentials of the spatial pattern. Although relaxation projections can also be devised for large-scale patterns (Ellner et al. 1998), in this chapter we focus attention on techniques suitable for understanding the implications of small-scale spatial heterogeneity.

\section{Correlation densities}

Given that interactions between individuals are local, the fate of each individual is determined by its local environment. To understand the ensuing dynamics, we must take a "plant's-eye view" (Turkington and Harper 1979) of spatial heterogeneity. If patterns are sufficiently small scale, individuals within each species experience similar "views," and essential degrees of freedom are thus given by descriptions of their average local environment. For example, Mahdi and Law (1987) measured average local environments in a community of grassland species by determining the expected densities of several other species at various radial distances around individuals of a focal species (see Figure 21.2a). We can do the same for the spatial patterns simulated in Figure 21.1b; results are shown in Figure 21.2b.

The quantities illustrated in Figure 21.2 are special cases of a general class of spatial statistics. These statistics are based on the densities at which certain spatial configurations appear in a given spatial pattern.

- The simplest spatial configuration is a singlet; the density of single individuals in a species across a given habitat simply corresponds to the mean density of the considered species. 
(a)

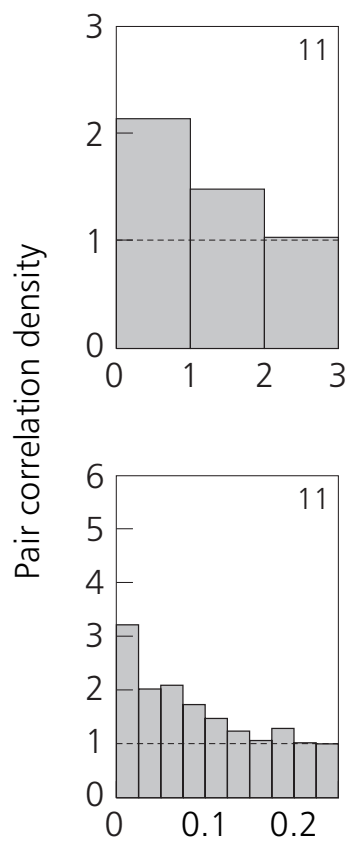

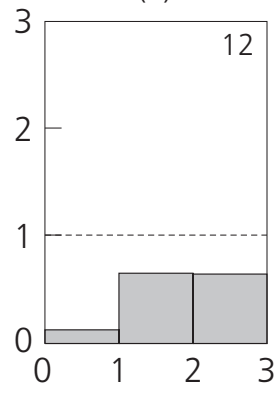

(b)

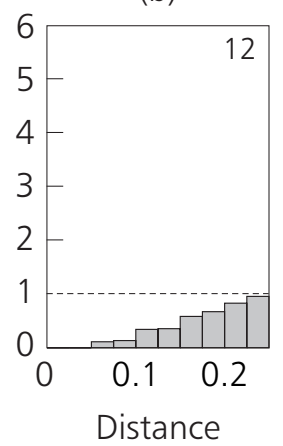

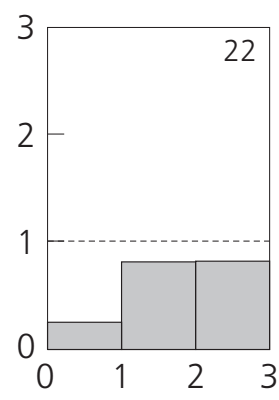

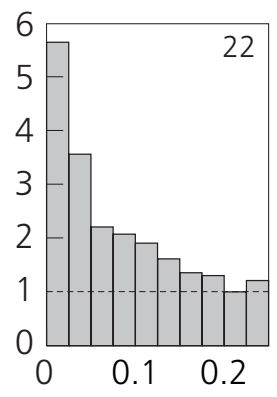

Figure 21.2 Pair correlation densities for field data and simulated pattern. Pair densities are normalized so that mean-field expectations are 1 (shown as dashed lines). (a) Measurements by Mahdi and Law (1987). Species 1 is Carex caryophyllea; species 2 is Carex flacca; distances are measured in $\mathrm{cm}$. At short distances, small cross-correlations indicate heterospecific segregation. (b) Pair densities for the spatial pattern in Figure 21.1b at time $t=15$. As can be verified by examining the original pattern, large auto-correlations and small cross-correlations suggest the existence of conspecific aggregations at spatial scales of 0.1 to 0.2 .

- Now consider the spatial density of pairs comprising an individual of species $i$ and one of species $j$ (where $j$ can be equal to $i$ ) that are a vectorial distance $\xi$ apart (see Figures 21.3a and 21.3b). Pair densities thus give information about the average local environments of individuals in each species. Local environments often are isotropic, that is, there are no special directions in space along which the pattern is aligned. In such cases (as in Figure 21.2), it is convenient to construct angular averages of pair densities, or, equivalently, to determine the spatial density of pairs that are a given length $r=|\xi|$ apart (see Figures 21.3c and 21.3d).

- Apart from pairs, we can also examine spatial configurations comprising three individuals; for instance, individuals of species $j$ and $k$ that are at distances $\xi$ and $\xi^{\prime}$, respectively, from an individual of species $i$. In an isotropic setting, these triangular configurations are characterized by three radii, $r, r^{\prime}$, and $r^{\prime \prime}=\left|\xi^{\prime}-\xi\right|$, corresponding to the three edges of the spanned triangle. 
(a)

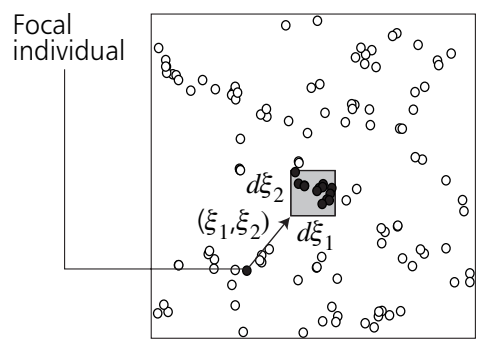

(b)

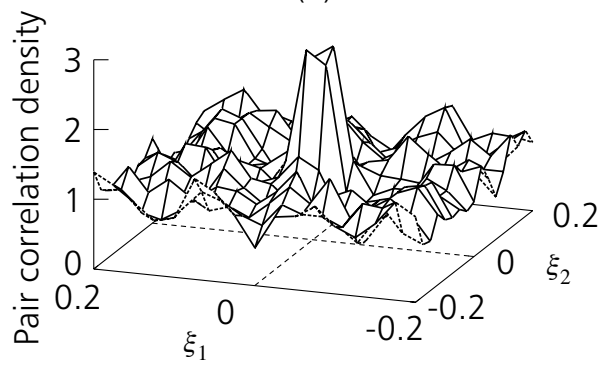

(c)

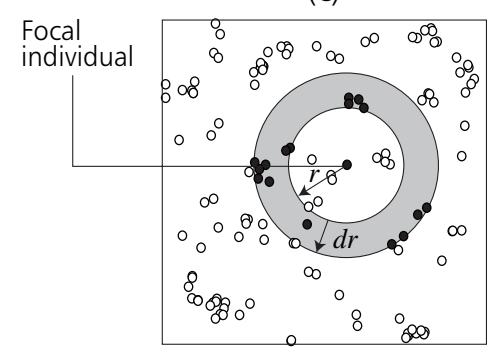

(d)

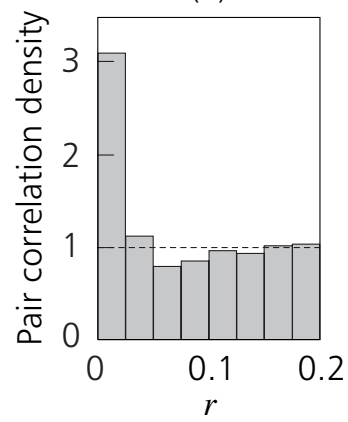

Figure 21.3 Measuring pair correlation densities. (a) For a given planar pattern of area $A$ and mean density $N$, the density of pairs at distance $\xi=\left(\xi_{1}, \xi_{2}\right)$ is assessed by counting, for a focal individual at location $x$, the number of paired individuals within the square $\left(x_{1}+\xi_{1}, x_{1}+\xi_{1}+d \xi_{1}\right) \times\left(x_{2}+\xi_{2}, x_{2}+\xi_{2}+d \xi_{2}\right)$, using a sufficiently small spatial resolution $d \xi$. Repeating this procedure with each individual in turn being the focal one and dividing the total count by $A$ and by $d \xi_{1} d \xi_{2}$ yields the pair density at distance $\xi$. Figure (b) illustrates how the resulting pair densities depend on $\xi$. (c) For isotropic patterns, it is useful to average pair densities over all angles of $\xi$. Equivalently, paired individuals are counted in rings of radius $r=|\xi|$ and width $d r$, centered on a focal individual. Total counts over all focal individuals are divided by $A$ and by $2 \pi r d r$. Figure (d) shows the resulting radial pair density. Pair densities in (b) and (d) are normalized by division through $N^{2}$ so that mean-field expectations are 1.

For any given pattern $p$ we can thus define correlation densities of order $m$ by

$$
C_{i_{1} \ldots i_{m}}\left(\xi_{1}, \ldots, \xi_{m-1}, p\right)=\frac{1}{A} \int p_{i_{1}}(x) \prod_{j=2}^{m} p_{i_{j}}\left(x+\xi_{j-1}\right) d x,
$$

where $i_{1}$ to $i_{m}$ specify the species of the $m$ individuals constituting the spatial configuration, and $\xi_{1}$ to $\xi_{m-1}$ denote the distances of individuals 2 to $m$, respectively, from individual 1 . The integration extends over all locations $x$ in the area $A$, which is assumed to be large enough that effects resulting from its boundary are negligible. As described above, special cases of correlation densities are given by mean densities (also referred to as global or singlet densities),

$$
C_{i}(p)=\frac{1}{A} \int p_{i}(x) d x,
$$


by pair densities (also referred to as local, environs, or doublet densities),

$$
C_{i j}(\xi, p)=\frac{1}{A} \int p_{i}(x) p_{j}(x+\xi) d x,
$$

and by triplet densities,

$$
C_{i j k}\left(\xi, \xi^{\prime}, p\right)=\frac{1}{A} \int p_{i}(x) p_{j}(x+\xi) p_{k}\left(x+\xi^{\prime}\right) d x .
$$

All correlation densities are given as integrals over products of spatial density functions and are therefore also known as spatial moments. Pair densities (second moments) $C_{i j}$ are also called auto-correlations for $i=j$ and cross-correlations for $i \neq j$. For mnemonic convenience, we denote mean densities (first moments) by $N_{i}=C_{i}$ and triplet densities (third moments) by $T_{i j k}=C_{i j k}$.

The spatial pattern $p$ of an ecological community comprising $m$ individuals is completely characterized (apart from its absolute location and orientation) by a correlation density of order $m$. This is because for two individuals the pair correlation density has a single peak at a location given by the vectorial distance between the two individuals. For three individuals, the triplet correlation function would have such a single peak at a location determined by the distances between individuals in the triangular configuration. Analogously, for $m$ individuals, information about all relative locations of individuals is available from the $m$ th-order correlation density. Although it is difficult to measure correlation densities of high order, it is evident that information about a spatial pattern can be represented either in location-based form (by specifying a pattern $p$ ) or in correlation-based form (by specifying a correlation density $C$ of sufficient order). These two representations contain exactly the same information about the relative position of all individuals. We can regard this equivalence as a coordinate transformation, expressing the same information in two different ways.

What, then, is the advantage of describing the dynamics of spatial patterns in correlation-based form? There are several reasons for doing so; here we give a preview of features that will be developed in this chapter.

- Quantitative descriptions of spatial patterns ought to be based on suitable summary statistics; correlation densities define such statistics. In particular, this class of statistics includes both mean densities and pair densities, two statistics that are natural for describing small-scale patterns and are readily applied to field data (Ripley 1981; Diggle 1983). Therefore, the expected dynamics of low-order correlation densities provide valuable insight into the main characteristics of a developing spatial process. 
- Because first-order correlations correspond to mean densities, meanfield models can be envisaged as a subset of correlation-based models. Correlation densities thus offer a systematic way of gradually extending and refining the set of summary statistics by successive integration of higher correlation orders. Such a task would be more difficult if based on sets of disparate types of spatial statistics.

- High-order correlations often relax much faster than low-order correlations (see Figure 21.4); consequently, essential degrees of freedom are captured by the low-order end of the correlation spectrum. It is for this reason that, for many spatio-temporal processes, relaxation manifolds (see Box 21.1) take a simple and tractable form in the space of correlation densities, whereas these manifolds tend to have a complicated topology in the space of spatial patterns or when expressed in terms of other spatial statistics. This feature of correlation densities greatly facilitates the application of relaxation projections.

- Pair densities naturally arise when assessing population-level consequences of pairwise interactions under spatial heterogeneity. In fact, the integrals over pair densities in Equations (21.1a) and (21.1c) extend the principle of "mass action" to spatially heterogeneous settings: formally speaking, individuals respond to linear functionals of spatial distributions.

- Whereas essential degrees of freedom in large-scale patterns can vary widely from process to process, correlation densities offer a universal representation of these essential degrees of freedom for small-scale patterns. A general theory of small-scale heterogeneity in ecological processes can therefore be built on the basis of correlation densities.

\section{Corrected correlation densities}

A simple modification of the correlation densities defined above is often helpful. Whenever a spatial configuration of order $m>1$ is considered, the same individual may figure twice in the description of the configuration. Whereas formally it is correct to count pairs that individuals form with themselves, from an ecological point of view such pairs are meaningless and misleading. For this reason, it is useful to construct corrected correlation densities, from which so-called self-pairs and similar repetitive configurations are removed. Corrected pair densities are marked by a tilde and, corresponding to Equation (21.3c) for uncorrected pair densities, are defined by

$$
\tilde{C}_{i j}(\xi, p)=\frac{1}{A} \int p_{i}(x)\left[p_{j}(x+\xi)-\delta_{i j} \delta_{x}(x+\xi)\right] d x .
$$


(a)
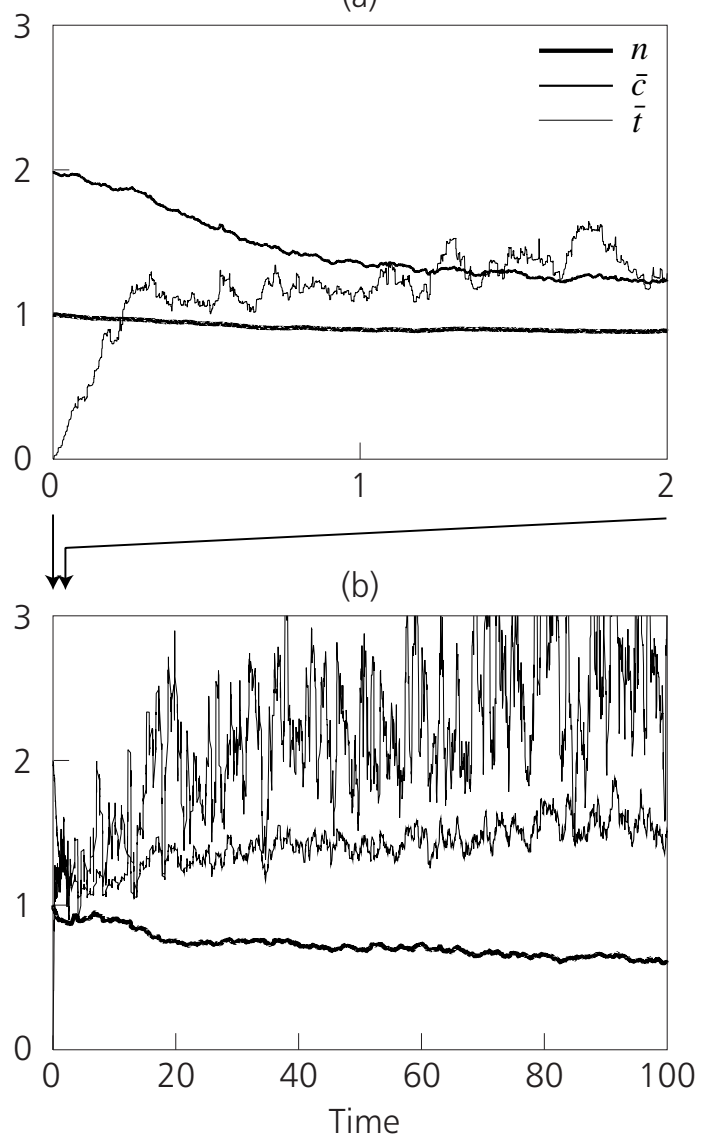

Figure 21.4 Relaxation time scales of correlation densities. Already for a singlespecies system, dynamics of pair and triplet densities cannot be depicted in full. We thus use integral statistics $\bar{c}=N_{1}^{-2} \int w_{11}^{(d)}(\xi) \tilde{C}_{11}(\xi) d \xi$ and $\bar{t}=N_{1}^{-3} \iint w_{11}^{(d)}(\xi) w_{11}^{(d)}\left(\xi^{\prime}\right)$ $\times \tilde{T}_{111}\left(\xi, \xi^{\prime}\right) d \xi d \xi^{\prime}$ instead and, for convenience, also normalize mean densities to $n=$ $N_{1} / N_{1}(0)$. As an initial condition, we choose a distribution of individuals, designed to satisfy $\bar{t}=0$. Even in the reduced state space $(n, \bar{c}, \bar{t})$, the system's response to such an artificial state is evident from the figure: (a) fast relaxation at a very short time scale is followed by (b) subsequent changes occurring over a much longer time scale. To diminish the amount of noise, time series are averaged over 10 realizations. Parameters are the same as in Figure 21.1a, except for s.d. $w_{11}^{(d)}=0.015$ and s.d. $m_{1}^{(b)}=0.05$.

With this correction, the focal individual of the first density function at location $x$ is removed from the second density function evaluated at location $x+\xi$. Of course, such removal is only necessary if the two density functions describe the same species; otherwise, individuals contributing to these functions belong to different species and therefore can never be identical; this is taken care of by the Kronecker symbol $\delta_{i j}$. Similarly, we can remove self-pairs and self-triplets from the third-order correlation densities,

$$
\begin{aligned}
\tilde{T}_{i j k}\left(\xi, \xi^{\prime}, p\right) & =\frac{1}{A} \int p_{i}(x)\left[p_{j}(x+\xi)-\delta_{i j} \delta_{x}(x+\xi)\right] \\
& \times\left[p_{k}\left(x+\xi^{\prime}\right)-\delta_{i k} \delta_{x}\left(x+\xi^{\prime}\right)-\delta_{j k} \delta_{x+\xi}\left(x+\xi^{\prime}\right)\right] d x
\end{aligned}
$$


where, for the third density function, we have to subtract contributions of the focal individuals of both the first and second density functions.

With this additional refinement in place, we can now turn to describing the dynamics of spatial patterns in terms of the corresponding dynamics of corrected correlation densities.

\section{Correlation dynamics}

Whenever a stochastic process of pattern dynamics is run, it creates a different realization (see, for example, Figure 14.2). This is in keeping with field observations which show that, although we may try to generate very similar initial spatial setups in, for example, plots of plants intended as replicates, the actual processes in each plot usually result in a wide variety of spatial patterns at later points in time. To extract the deterministic signal of a spatial ecological process from among all accompanying stochastic noise, averages over replicates are needed. If, however, we were to average spatial density functions across the many different patterns that can arise from a single initial condition, we would merely obtain an uninformative and rather unstructured "average pattern": in the long run, high abundances at some location in one replicate would cancel out low abundances at the same location in other replicates. It is therefore important to average over spatial statistics, rather than across spatial patterns. For example, the mean density of a particular species averaged across realizations is a meaningful ecological quantity.

In this vein, we define expected values of corrected correlation functions as

$$
\tilde{C}_{i_{1} \ldots i_{m}}\left(\xi_{1}, \ldots, \xi_{m-1}\right)=\int \tilde{C}_{i_{1} \ldots i_{m}}\left(\xi_{1}, \ldots, \xi_{m-1}, p\right) P(p) d p
$$

that is, by weighting the correlation densities for a pattern $p$ with the probability density $P(p)$ for that pattern to occur. We can now study the dynamics of expected correlation densities [for notational convenience we omit the arguments $\left(\xi_{1}, \ldots, \xi_{m-1}\right)$ of $\tilde{C}_{i_{1} \ldots i_{m}}$ in the next three equations],

$$
\frac{d}{d t} \tilde{C}_{i_{1} \ldots i_{m}}=\int \tilde{C}_{i_{1} \ldots i_{m}}(p) \frac{d}{d t} P(p) d p .
$$

Using Equation (21.2a), this gives

$$
\frac{d}{d t} \tilde{C}_{i_{1} \ldots i_{m}}=\int \tilde{C}_{i_{1} \ldots i_{m}}(p) \int\left[w\left(p \mid p^{\prime}\right) P\left(p^{\prime}\right)-w\left(p^{\prime} \mid p\right) P(p)\right] d p^{\prime} d p
$$

which, by separating the difference into two integrals, swapping the integration variables $p$ and $p^{\prime}$ in the first of them, and then joining the two 
integrals again, yields

$\frac{d}{d t} \tilde{C}_{i_{1} \ldots i_{m}}=\int\left\{\int\left[\tilde{C}_{i_{1} \ldots i_{m}}\left(p^{\prime}\right)-\tilde{C}_{i_{1} \ldots i_{m}}(p)\right] w\left(p^{\prime} \mid p\right) d p^{\prime}\right\} P(p) d p$. (21.6c)

The term in curly braces is known as the first jump moment (van Kampen 1992) of the $m$ th-order correlation density; it describes the correlation density's expected rate of change around a given pattern $p$, and we denote it by $a_{i_{1} \ldots i_{m}}\left(\xi_{1}, \ldots, \xi_{m-1}, p\right)$.

\section{Dynamics of mean densities}

We can now evaluate the expected rate of change in mean densities, or first spatial moments, by using Equations (21.2b) and (21.3b) in Equation (21.6c). According to Equation (21.2b), $a_{i}(p)$, the first jump moment of the first spatial moment in species $i$, can be decomposed into three contributions coming from birth, death, and movement events, respectively,

$$
a_{i}(p)=a_{i}^{(b)}(p)+a_{i}^{(d)}(p)+a_{i}^{(m)}(p) .
$$

The birth contribution is

$$
\begin{aligned}
a_{i}^{(b)}(p) & =\int\left[N_{i}\left(p^{\prime}\right)-N_{i}(p)\right] \\
& \times \sum_{j} \iint B_{j}\left(x, x^{\prime}, p\right) p_{j}(x) \Delta\left(p+u_{j} \delta_{x^{\prime}}-p^{\prime}\right) d x d x^{\prime} d p^{\prime},
\end{aligned}
$$

which, after collapsing the integration over $p^{\prime}$ using the definition of the generalized delta function (see Box 21.4), simplifies to

$a_{i}^{(b)}(p)=\sum_{j} \iint\left[N_{i}\left(p+u_{j} \delta_{x^{\prime}}\right)-N_{i}(p)\right] B_{j}\left(x, x^{\prime}, p\right) p_{j}(x) d x d x^{\prime}$.

For all $j \neq i$, the term in square brackets is 0 , while for $j=i$ it is $\frac{1}{A}$. We see this by considering $N_{i}\left(p+u_{j} \delta_{x^{\prime}}\right)=\frac{1}{A} \int\left(p+u_{j} \delta_{x^{\prime}}\right)_{i}(x) d x=$ $\frac{1}{A} \int p_{i}(x) d x+\frac{1}{A} \delta_{i j} \int \delta_{x^{\prime}}(x) d x=N_{i}(p)+\frac{1}{A} \delta_{i j}$. This gives

$$
a_{i}^{(b)}(p)=\frac{1}{A} \iint B_{i}\left(x, x^{\prime}, p\right) p_{i}(x) d x d x^{\prime},
$$

or, by using Equation (21.1c) and exploiting that $m_{i}^{(b)}$ is the probability density for dispersal and thus integrates to 1 ,

$$
\begin{aligned}
a_{i}^{(b)}(p) & =\frac{1}{A} \int\left[b_{i}+\sum_{j} b_{i j}^{\prime} \int w_{i j}^{(b)}\left(x^{\prime \prime}-x\right)\right. \\
& \left.\times\left[p_{j}\left(x^{\prime \prime}\right)-\delta_{i j} \delta_{x}\left(x^{\prime \prime}\right)\right] d x^{\prime \prime}\right] p_{i}(x) d x,
\end{aligned}
$$


or, when separating terms for density-independent and density-dependent birth,

$$
\begin{aligned}
a_{i}^{(b)}(p)= & b_{i} \frac{1}{A} \int p_{i}(x) d x+\sum_{j} b_{i j}^{\prime} \frac{1}{A} \iint w_{i j}^{(b)}\left(x^{\prime \prime}-x\right) \\
& \times p_{i}(x)\left[p_{j}\left(x^{\prime \prime}\right)-\delta_{i j} \delta_{x}^{\prime}\left(x^{\prime \prime}\right)\right] d x d x^{\prime \prime} .
\end{aligned}
$$

We now replace the integration over $x$ with one over $\xi=x^{\prime \prime}-x$, use the definitions of $N_{i}(p)$ and $\tilde{C}_{i j}(\xi, p)$, and thus obtain

$$
a_{i}^{(b)}(p)=b_{i} N_{i}(p)+\sum_{j} b_{i j}^{\prime} \int w_{i j}^{(b)}(\xi) \tilde{C}_{i j}(\xi, p) d \xi .
$$

For death events, we have $N_{i}\left(p-u_{j} \delta_{x}\right)-N_{i}(p)=-\frac{1}{A} \delta_{i j}$, which yields

$$
a_{i}^{(d)}(p)=-d_{i} N_{i}(p)-\sum_{j} d_{i j}^{\prime} \int w_{i j}^{(d)}(\xi) \tilde{C}_{i j}(\xi, p) d \xi
$$

while $N_{i}\left(p-u_{j} \delta_{x}+u_{j} \delta_{x^{\prime}}\right)-N_{i}(p)=0$ for movement events gives

$$
a_{i}^{(m)}(p)=0 \text {. }
$$

Collecting all three results together, and using Equations (21.6c) and (21.7), gives the expected rate of change in the mean density of species $i$

$$
\begin{aligned}
\frac{d}{d t} N_{i}= & +b_{i} N_{i}+\sum_{j} b_{i j}^{\prime} \int w_{i j}^{(b)}(\xi) \tilde{C}_{i j}(\xi) d \xi \\
& -d_{i} N_{i}-\sum_{j} d_{i j}^{\prime} \int w_{i j}^{(d)}(\xi) \tilde{C}_{i j}(\xi) d \xi .
\end{aligned}
$$

This result is readily interpreted. Movement does not contribute to the dynamics of mean densities at all: moving individuals around does not alter their abundance. Notice that, according to Equation (21.9), for both birth and death events the interaction kernels $w_{i j}^{(b)}(\xi)$ and $w_{i j}^{(d)}(\xi)$ of individuals of species $j$ at distance $\xi$ from individuals in species $i$, are weighted with the density $\tilde{C}_{i j}(\xi)$ at which individuals of species $j$ are expected to be found at a distance $\xi$ around individuals of species $i$. Effective local densities - that is, the integrals in Equation (21.9) - are then simply the sum of all such contributions. In this way, the mean densities within a spatial pattern change according to the "individual's-eye view" of its local environment, as described by the pair densities. If spatial heterogeneity were removed by setting $\tilde{C}_{i j}(\xi)=N_{i} N_{j}$ for all $i, j$, and $\xi$, we would recover the familiar Lotka-Volterra dynamics of mean-field theory, $\frac{d}{d t} N_{i}=\left(b_{i}-d_{i}\right) N_{i}+\sum_{j}\left(b_{i j}^{\prime}-d_{i j}^{\prime}\right) N_{i} N_{j}$. For spatially heterogeneous systems, however, this simple description is incorrect and the dynamics of mean densities become contingent on those of pair densities. 


\section{Dynamics of pair densities}

To provide the information on corrected pair densities $\tilde{C}_{i j}(\xi)$ as required in Equation (21.9), we need to work out their expected rates of change. For this purpose, we follow the same sequence of steps as was used for deriving the dynamics of mean densities and thereby gain the following general result:

$$
\frac{d}{d t} \tilde{C}_{i j}(\xi)=
$$$$
\operatorname{Birth}\left\{\begin{array}{l}
+\delta_{i j} b_{i} m_{i}^{(b)}(-\xi) N_{i} \\
+b_{i} \int m_{i}^{(b)}\left(\xi^{\prime}\right) \tilde{C}_{i j}\left(\xi+\xi^{\prime}\right) d \xi^{\prime} \\
+\delta_{i j} m_{i}^{(b)}(-\xi) \sum_{k} b_{i k}^{\prime} \int w_{i k}^{(b)}\left(\xi^{\prime \prime}\right) \tilde{C}_{i k}\left(\xi^{\prime \prime}\right) d \xi^{\prime \prime} \\
+\sum_{k} b_{i k}^{\prime} \iint m_{i}^{(b)}\left(\xi^{\prime}\right) w_{i k}^{(b)}\left(\xi^{\prime \prime}\right) \tilde{T}_{i j k}\left(\xi+\xi^{\prime}, \xi^{\prime \prime}\right) d \xi^{\prime} d \xi^{\prime \prime} \\
+b_{i j}^{\prime} \int m_{i}^{(b)}\left(\xi^{\prime}\right) w_{i j}^{(b)}\left(\xi+\xi^{\prime}\right) \tilde{C}_{i j}\left(\xi+\xi^{\prime}\right) d \xi^{\prime}
\end{array}\right.
$$$$
\text { Death }\left\{\begin{array}{l}
-d_{i} \tilde{C}_{i j}(\xi) \\
-\sum_{k} d_{i k}^{\prime} \int w_{i k}^{(d)}\left(\xi^{\prime \prime}\right) \tilde{T}_{i j k}\left(\xi, \xi^{\prime \prime}\right) d \xi^{\prime \prime} \\
-d_{i j}^{\prime} w_{i j}^{(d)}(\xi) \tilde{C}_{i j}(\xi)
\end{array}\right.
$$

$$
\text { Movement }\left\{\begin{array}{l}
-\left|m_{i}\right| \tilde{C}_{i j}(\xi) \\
+\int m_{i}\left(\xi^{\prime}\right) \tilde{C}_{i j}\left(\xi+\xi^{\prime}\right) d \xi^{\prime} \\
+\langle i, j, \xi \rightarrow j, i,-\xi\rangle .
\end{array}\right.
$$

The derivation of this equation is provided in Appendix 21.A. All terms on the right-hand side have a precise interpretation, given below. We first focus on the $i$ individual of the $i j$ pair; analogous considerations apply to events undergone by the $j$ individual, as discussed at the end of the section.

The first five terms on the right-hand side describe the increase in the density of $i j$ pairs at vectorial distance $\xi$ resulting from birth events in species $i$. 
- A new $i$ individual, arising from density-independent birth, generates a parent-offspring pair of type $i i$ with its parent. This is accounted for by the first term, which therefore only contributes to the expected dynamics of auto-correlation densities; this is reflected by the Kronecker delta $\delta_{i j}$. Multiplying the mean density of individuals $N_{i}$ by the densityindependent per capita birth rate $b_{i}$ gives the rate for such events creating a pair at any distance $\xi$; different distances occur at spatial density $m_{i}^{(b)}(-\xi)$ of parents around dispersed offspring.

- The second term also accounts for density-independent birth, but focuses on the new pair that the $i$ offspring forms with the $j$ neighbor of its parent. If the $i$ individual of an $i j$ pair at distance $\xi+\xi^{\prime}$ gives birth to a new individual at distance $\xi^{\prime}$ from its parent, a new $i j$ pair at distance $\xi$ is generated. The per capita rate for density-independent birth is $b_{i}$, the density of $i j$ pairs at distance $\xi+\xi^{\prime}$ is $\tilde{C}_{i j}\left(\xi+\xi^{\prime}\right)$, and the spatial density of offspring settling around the $i$ parent is $m_{i}^{(b)}\left(\xi^{\prime}\right)$. Multiplying these three factors and integrating over all possible distances $\xi^{\prime}$ of offspring dispersal yields the second term.

- The third term describes how, under density-dependent birth, a new parent-offspring pair changes the density of $i i$ pairs. Fecundity of an $i$ individual can be modified by the presence of a $k$ individual at distance $\xi^{\prime \prime}$. The expected density of $k$ individuals around the $i$ individual is $\tilde{C}_{i k}\left(\xi^{\prime \prime}\right)$, the interaction kernel for the $i k$ pair yields $w_{i k}^{(b)}\left(\xi^{\prime \prime}\right)$, so that integrating over all interaction distances $\xi^{\prime \prime}$ gives the third term's integral. We then have to sum over all species $k$ that exert such an effect: sign and weight for the contribution of each species are determined by coefficients $b_{i k}^{\prime}$. The resulting density-dependent per capita birth rate is multiplied by $\delta_{i j}$, since this term only affects the dynamics of autocorrelations, and by $m_{i}^{(b)}(-\xi)$, the density of parent individuals as seen from the $i$ offspring.

- The fourth term describes the effect of new offspring-neighbor pairs of type $i j$ arising from density-dependent birth. As in the previous term, the fecundity of an $i$ individual in an $i j$ pair at distance $\xi+\xi^{\prime}$ can be modified by the presence of a $k$ individual at distance $\xi^{\prime \prime}$ relative to the $i$ individual. Under these circumstances, an $i$ offspring, located at distance $\xi^{\prime}$ from its parent, generates a new $i j$ pair at distance $\xi$. The density of the original $i j k$ triplet configuration is $\tilde{T}_{i j k}\left(\xi+\xi^{\prime}, \xi^{\prime \prime}\right)$, the interaction kernel for the $i k$ pair yields $w_{i k}^{(b)}\left(\xi^{\prime \prime}\right)$, and the spatial density of offspring around parent individuals is $m_{i}^{(b)}\left(\xi^{\prime}\right)$. Multiplying these factors and integrating over all possible dispersal distances $\xi^{\prime}$ and interaction distances 
$\xi^{\prime \prime}$ weights the effect of species $k$ in the fourth term's sum. The resulting integrals are summed over all species $k$ that exert such an effect on $i$ 's fecundity: signs and weights for these contributions are again determined by the interaction coefficients $b_{i k}^{\prime}$.

- The fifth term, the last to account for birth effects, describes the consequences of density-dependence of birth rates within the $i j$ pair. The density of such pairs at distance $\xi+\xi^{\prime}$ is $\tilde{C}_{i j}\left(\xi+\xi^{\prime}\right)$, and the effect that interaction with $j$ has on $i$ 's birth rate is given by $b_{i j}^{\prime} w_{i j}^{(b)}\left(\xi+\xi^{\prime}\right)$. An $i$ offspring at distance $\xi^{\prime}$ from the $i$ parent, arising at density $m_{i}^{(b)}\left(\xi^{\prime}\right)$, creates a new $i j$ pair at distance $\xi$. Integrating these three factors over all dispersal distances $\xi^{\prime}$ yields the fifth term.

The next three terms account for the decrease in the density of $i j$ pairs at distance $\xi$ due to death events in species $i$.

- Density-independent death of the $i$ individual of the $i j$ pair occurs at per capita rate $d_{i}$. Multiplying $d_{i}$ by the density $\tilde{C}_{i j}(\xi)$ of $i j$ pairs at distance $\xi$ in the sixth term gives the resulting decrease in that density.

- Density-dependent death of the $i$ individual of the $i j$ pair can result from the presence of a $k$ individual at distance $\xi^{\prime \prime}$. Such triplet configurations occur at density $\tilde{T}_{i j k}\left(\xi, \xi^{\prime \prime}\right)$, and the cumulative effect of individuals of species $k$, situated at different distances from the $i$ individual, is obtained by weighting the triplet density with $w_{i k}^{(d)}\left(\xi^{\prime \prime}\right)$, given by the interaction kernel for density-dependent death, and integrating the product over all interaction distances $\xi^{\prime \prime}$. Multiplying this contribution of species $k$ with the corresponding interaction coefficient $d_{i k}^{\prime}$ and summing over all species $k$ gives the seventh term.

- Like birth events, the density-dependent component of death rates can also be affected by interaction of the focal $i$ individual with its $j$ partner within the $i j$ pair. The interaction coefficient for this interaction is $d_{i j}^{\prime}$, the effect of distance $\xi$ on the strength of interaction is measured by $w_{i j}^{(d)}(\xi)$, and the pair configuration occurs at density $\tilde{C}_{i j}(\xi)$. The product of these three factors gives the eighth term.

The next two terms describe how the density of $i j$ pairs at distance $\xi$ changes because of movement of the $i$ individual.

- When the $i$ individual moves, the original $i j$ pair at distance $\xi$ is destroyed. The ninth term reflects this: here, the per capita rate $\left|m_{i}\right|$ for $i$ 's movement is multiplied by the density $\tilde{C}_{i j}(\xi)$ of the original pair configuration. 
- On the other hand, a new $i j$ pair at distance $\xi$ is created when the original pair distance is $\xi+\xi^{\prime}$ and the $i$ individual moves a distance $\xi^{\prime}$. This effect is captured by the tenth term, which weights the density $\tilde{C}_{i j}\left(\xi+\xi^{\prime}\right)$ of the original pair configuration with the movement kernel $m_{i}(\xi)$ for species $i$ and integrates over all possible movement distances $\xi^{\prime}$.

In all events described above we have focused on the $i$ individual. Of course, analogous events can occur to the $j$ individual of the $i j$ pair. When swapping the roles of the $i$ and $j$ individuals, the distance vector $\xi$ must also be reversed. The last term $\langle i, j, \xi \rightarrow j, i,-\xi\rangle$ accounts for this: it is shorthand for all preceding terms after changing $i$ to $j, j$ to $i$, and $\xi$ to $-\xi$.

\subsection{Moment Closures and their Performance}

A critical step toward simplifying the spatial complexity of ecological birth-death-movement models is to close the correlation dynamics derived in Equations (21.9) and (21.10). In this section, we introduce alternative closures and investigate their relative performance.

\section{Moment hierarchies and moment closures}

While the right-hand side of the mean densities' dynamics, Equation (21.9), depends on pair densities, that of the pair densities' dynamics, Equation (21.10), contains triplet densities. This observation can be generalized: when interactions are pairwise, for any order $m$ of correlation densities, dynamics are contingent on correlation densities of order $m+1$. The resulting sequence of equations is called a moment hierarchy, the head of which is given by Equations (21.9) and (21.10). This hierarchy precludes exploiting the dynamics of $N$ unless we have information on $\tilde{C}$; this information comes from the dynamics of $\tilde{C}$, for which we need information on $\tilde{T}$, and so on.

To escape from this cascade of dependencies, the moment hierarchy has to be truncated. In other words, we have to express the correlation densities of order $m+1$ in terms of those of order $m$ and below. Such expressions are called moment closures. In the previous section, we saw that inserting

$$
\tilde{C}_{i j}(\xi)=N_{i} N_{j}
$$

into Equation (21.9) yields a closed dynamical system that is the meanfield approximation of the considered birth-death-movement process. This comes as no surprise: assuming $\tilde{C}_{i j}(\xi)=N_{i} N_{j}$ for all $\xi$ implies pair densities that are independent of distance or, equivalently, the absence of 
salient spatial structure. These are precisely the circumstances under which the mean-field assumption is valid. We therefore conclude that using the moment closure given in Equation (21.11) to truncate the moment hierarchy after the first order recovers the mean-field approximation.

To improve on this result we go beyond the first order of the hierarchy: the natural next step is to truncate the hierarchy after its second order. For this purpose, we have to express triplet densities, or third moments $\tilde{T}$, in terms of first and second moments $N$ and $\tilde{C}$. Notice that in the derivation of Equations (21.9) and (21.10) no approximations have been introduced; consequently, these equations are exact. The price that has to be paid for a moment closure is that the resulting description is an approximation. In fact, the performance of such an approximation may vary with the moment closure chosen. So what criteria can guide the choice of moment closures to give a good approximation and the desired truncation of the moment hierarchy at the second order?

\section{Conditions for moment closures}

Moment closures are not uniquely determined and thus alternative versions can be chosen. Yet, some conditions narrow the range of options.

- Condition (C1). In the absence of any pair correlations, no information on spatial structure is available to closures for triplet densities, and therefore individuals in triplets must also be assumed to be uncorrelated. In other words, if $\tilde{C}_{i j}(\xi)=N_{i} N_{j}$ for all $i, j$, and $\xi$, any consistent moment closure ought to yield $\tilde{T}_{i j k}\left(\xi, \xi^{\prime}\right)=N_{i} N_{j} N_{k}$ for all $i, j, k, \xi$, and $\xi^{\prime}$.

- Condition (C2). Because attention is focused on small-scale spatial structure, pairs of individuals separated by large distances are assumed to be uncorrelated. Therefore, $\lim _{|\xi| \rightarrow \infty} \tilde{C}_{i j}(\xi)=N_{i} N_{j}$ and $\lim _{|\xi| \rightarrow \infty} \frac{d}{d t} \tilde{C}_{i j}(\xi)=\frac{d}{d t}\left(N_{i} N_{j}\right)=N_{i} \frac{d}{d t} N_{j}+N_{j} \frac{d}{d t} N_{i}$ for all $i$ and $j$, hold in general: any potentially developing structure is supposed not to affect pair densities at large distances. When evaluating Equation (21.10) in this limit, consistency with Equation (21.9) requires that we assume (i) $\lim _{|\xi| \rightarrow \infty} \int w_{i k}^{(d)}\left(\xi^{\prime \prime}\right) \tilde{T}_{i j k}\left(\xi, \xi^{\prime \prime}\right) d \xi^{\prime \prime}=$ $N_{j} \int w_{i k}^{(d)}\left(\xi^{\prime \prime}\right) \tilde{C}_{i k}\left(\xi^{\prime \prime}\right) d \xi^{\prime \prime}$ and (ii) $\lim _{|\xi| \rightarrow \infty} \iint m_{i}^{(b)}\left(\xi^{\prime}\right) w_{i k}^{(b)}\left(\xi^{\prime \prime}\right) \tilde{T}_{i j k}(\xi+$ $\left.\xi^{\prime}, \xi^{\prime \prime}\right) d \xi^{\prime} d \xi^{\prime \prime}=N_{j} \int w_{i j}^{(b)}\left(\xi^{\prime \prime}\right) \tilde{C}_{i j}\left(\xi^{\prime \prime}\right) d \xi^{\prime \prime}$ for all $i, j$, and $k$, and for all kernels $w_{i k}^{(d)}, w_{i k}^{(b)}$, and $m_{i}^{(b)}$. Conditions (i) and (ii) are fulfilled if, and only if, $\lim _{|\xi| \rightarrow \infty} \tilde{T}_{i j k}\left(\xi, \xi^{\prime \prime}\right)=N_{j} \tilde{C}_{i k}\left(\xi^{\prime \prime}\right)$ holds for all $i, j, k$, and $\xi^{\prime \prime}$.

The two conditions above provide criteria for valid moment closures. We now have to consider how to characterize good moment closures. 


\section{Moment closures as relaxation projections}

To distinguish better moment closures from less suitable ones, the notion of relaxation projections (see Box 21.1) becomes critical. Usually, not all degrees of freedom in a system change on the same time scale. Some degrees of freedom are fast and decay quickly, while others are slower and thus remain essential for a longer time. In particular, triplet densities often have a much faster pace of change than both pair and mean densities. Figure 21.4 gives an example illustrating this feature.

After a system's fast degrees of freedom have decayed, they are no longer independent variables and instead become functions of the slower degrees of freedom. For example, when pair densities $\tilde{C}$ have decayed, it must become possible to express them in terms of mean densities $N$. Such a relation is provided by Equation (21.11); it truncates the moment hierarchy at first order. The mean-field approximation therefore can also be interpreted as a relaxation projection. Likewise, after triplet densities $\tilde{T}$ have decayed, they lose their role as essential degrees of freedom and can be expressed as functions of pair densities $\tilde{C}$ and mean densities $N$. Such relations are the moment closures we are seeking for truncating the moment hierarchy at second order. As described in Box 21.1, good moment closures match a system's relaxation manifold and define a projection onto this manifold that resembles the system's actual relaxation dynamics.

\section{Candidate moment closures}

To express triplet densities $\tilde{T}$ as a function of pair densities $\tilde{C}$ and mean densities $N$, different assumptions for the relaxation manifold can be made. Here we introduce and investigate four possible candidates:

$$
\begin{aligned}
\tilde{T}_{i j k}\left(\xi, \xi^{\prime}\right)= & \tilde{C}_{i j}(\xi) N_{k}+\tilde{C}_{i k}\left(\xi^{\prime}\right) N_{j} \\
& +\tilde{C}_{j k}\left(\xi^{\prime}-\xi\right) N_{i}-2 N_{i} N_{j} N_{k}, \\
\tilde{T}_{i j k}\left(\xi, \xi^{\prime}\right)= & \tilde{C}_{i j}(\xi) \tilde{C}_{i k}\left(\xi^{\prime}\right) / N_{i}, \\
\tilde{T}_{i j k}\left(\xi, \xi^{\prime}\right)= & \frac{1}{2}\left[\tilde{C}_{i j}(\xi) \tilde{C}_{i k}\left(\xi^{\prime}\right) / N_{i}\right. \\
& +\tilde{C}_{i j}(\xi) \tilde{C}_{j k}\left(\xi^{\prime}-\xi\right) / N_{j} \\
& \left.+\tilde{C}_{i k}\left(\xi^{\prime}\right) \tilde{C}_{j k}\left(\xi^{\prime}-\xi\right) / N_{k}-N_{i} N_{j} N_{k}\right], \\
& \tilde{C}_{i j}(\xi) \tilde{C}_{i k}\left(\xi^{\prime}\right) \tilde{C}_{j k}\left(\xi^{\prime}-\xi\right) /\left(N_{i} N_{j} N_{k}\right) .
\end{aligned}
$$




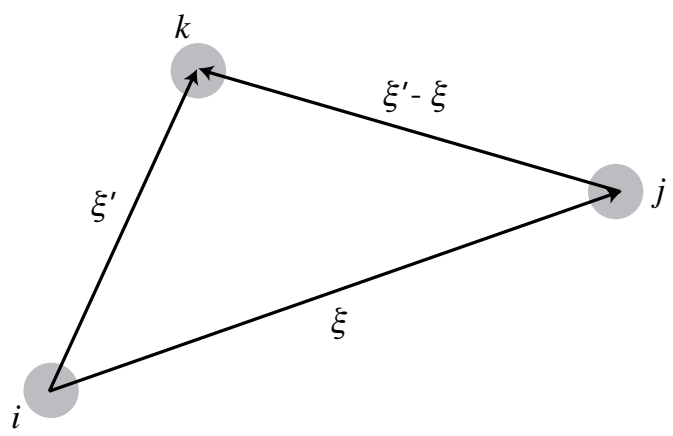

Figure 21.5 Triplet configuration for moment closure. Individuals of species $i, j$, and $k$ are separated by vectors $\xi, \xi^{\prime}$, and $\xi^{\prime}-\xi$.

All these candidate closures satisfy Conditions (C1) and (C2) as defined above. As a counterexample, consider an alternative candidate, $\tilde{T}_{i j k}\left(\xi, \xi^{\prime}\right)=\frac{1}{3}\left[\tilde{C}_{i j}(\xi) N_{k}+\tilde{C}_{i k}\left(\xi^{\prime}\right) N_{j}+\tilde{C}_{j k}\left(\xi^{\prime}-\xi\right) N_{i}\right]$, for which Condition (C1) holds but (C2) is violated.

The salient ingredients for these closures are the pair correlations along the three sides of the triangular configuration of the $i, j$, and $k$ individuals: $\tilde{C}_{i j}(\xi), \tilde{C}_{i k}\left(\xi^{\prime}\right)$, and $\tilde{C}_{j k}\left(\xi^{\prime}-\xi\right)$ (see Figure 21.5). The closures above can be constructed according to the following recipe.

- First, choose the power of the closure. This is the number of pair densities that are multiplied by each other and determines the "building blocks" of the closure: for a power-1 closure, each building block is a pair density; for a power- 2 closure it is the product of two pair densities; and for a power-3 closure, the product of three pair densities.

- Second, construct all building blocks arising from permutations among the three corners of the triangle.

- Third, modify each building block by multiplying or dividing by mean densities such that it satisfies Condition (C1).

- Fourth, add all blocks.

- Fifth, modify the sum by adding or subtracting $N_{i} N_{j} N_{k}$ blocks and divide by the resulting net number of blocks so that Condition $(\mathrm{C} 2)$ is met.

Closures in Equations (21.12a), (21.12c), and (21.12d) simply follow this recipe for powers 1,2 , and 3, respectively. Higher powers are not considered since they would require repeating pair densities in building blocks.

The closure in Equation (21.12b) is obtained from the same recipe by modifying the second step. This is motivated by the asymmetric way triplet densities enter into Equation (21.10): triplet densities there characterize the density of $j$ and $k$ individuals around a focal $i$ individual (or of $i$ and $k$ individuals around a focal $j$ individual, see below), whose birth and death rates 
are affected by its local surroundings. The $i$ individual therefore has a special role in the triplet; this is reflected in the closure in Equation (21.12b), which concentrates on the pair densities along those two edges of the $i j k$ triangle that are connected to the corner occupied by the $i$ individual (i.e., the $i j$ and $i k$ edges). For a focal $j$ individual, the same consideration applies: now the triplet configuration in Equation (21.10) is $j i k$, and in Equation (21.12b) the special role is assumed by the $j$ individual. The closure in Equation (21.12c), in contrast, gives equal weight to all three pairs of edges of the triangle; the resulting expression for $\tilde{T}_{i j k}$ is thus completely symmetric under permutations of the $i, j$, and $k$ individuals. Closures that reflect the special role of the one focal individual cannot be constructed for powers 1 and 3; thus, the asymmetric power- 2 closure, Equation (21.12b), is the only asymmetric closure included in the considered set of candidates.

The power-1 moment closure in Equation (21.12a) can be motivated by defining so-called central third moments, $T_{i j k}^{(c)}\left(\xi, \xi^{\prime}, p\right)=\frac{1}{A} \int\left[p_{i}(x)-\right.$ $\left.N_{i}\right]\left[p_{j}(x+\xi)-N_{j}\right]\left[p_{k}\left(x+\xi^{\prime}\right)-N_{k}\right] d x$, and is based on the assumption that these are vanishing (see Chapter 20; Bolker and Pacala 1997). In contrast, the motivation for power- 2 closures comes from a probabilistic argument. When assessing the probability density of a triangular configuration based on pair correlations along edges, we notice that only two of the triangle's three edge vectors can be chosen independently; the third edge vector directly follows from choosing the other two. Envisaging the probability density for a particular triangle as the joint probability density for two of its edge vectors and assuming that the contribution of both edges is statistically independent naturally leads to moment closures that use products of two pair densities as building blocks (see Chapter 14; Dieckmann et al. 1997, in press). The power-3 closure in Equation (21.12d) has a long tradition in the literature of theoretical physics, where it is known as the Kirkwood superposition approximation (Kirkwood 1935; Ziman 1979).

\section{Testing the candidate closures}

We now test the performance of the four candidate closures by comparing the results of individual-based simulations with predictions derived from these moment closures.

The first test considers triplet densities directly. This requires measuring, at one moment in time, the distribution of triplets in the three-dimensional space spanned by the edge lengths $\left(r, r^{\prime}, r^{\prime \prime}\right)$ of all triangles formed by triplets of individuals. For a triplet configuration with a pair $\left(\xi, \xi^{\prime}\right)$ of edge vectors, we have $r=|\xi|, r^{\prime}=\left|\xi^{\prime}\right|$, and $r^{\prime \prime}=\left|\xi^{\prime}-\xi\right|$ (see Figure 21.5). Contour surfaces resulting from such a measurement of triplet densities are 
(a)

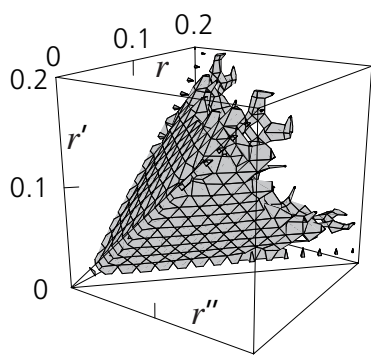

(b)

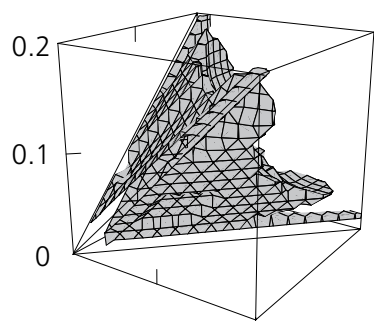

(c)

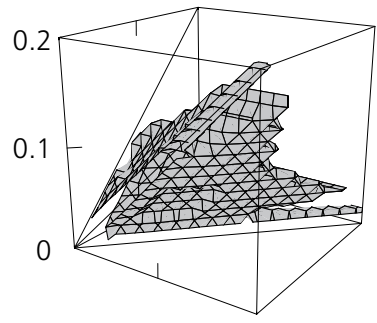

(d)

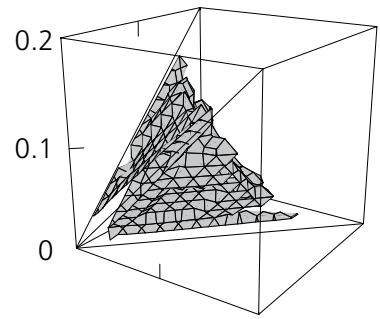

(e)

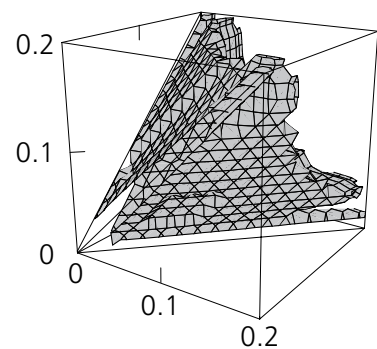

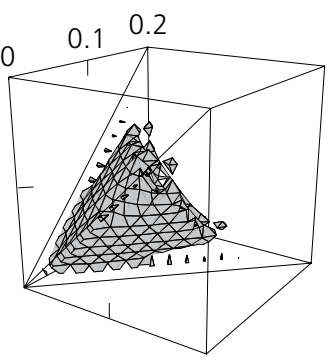
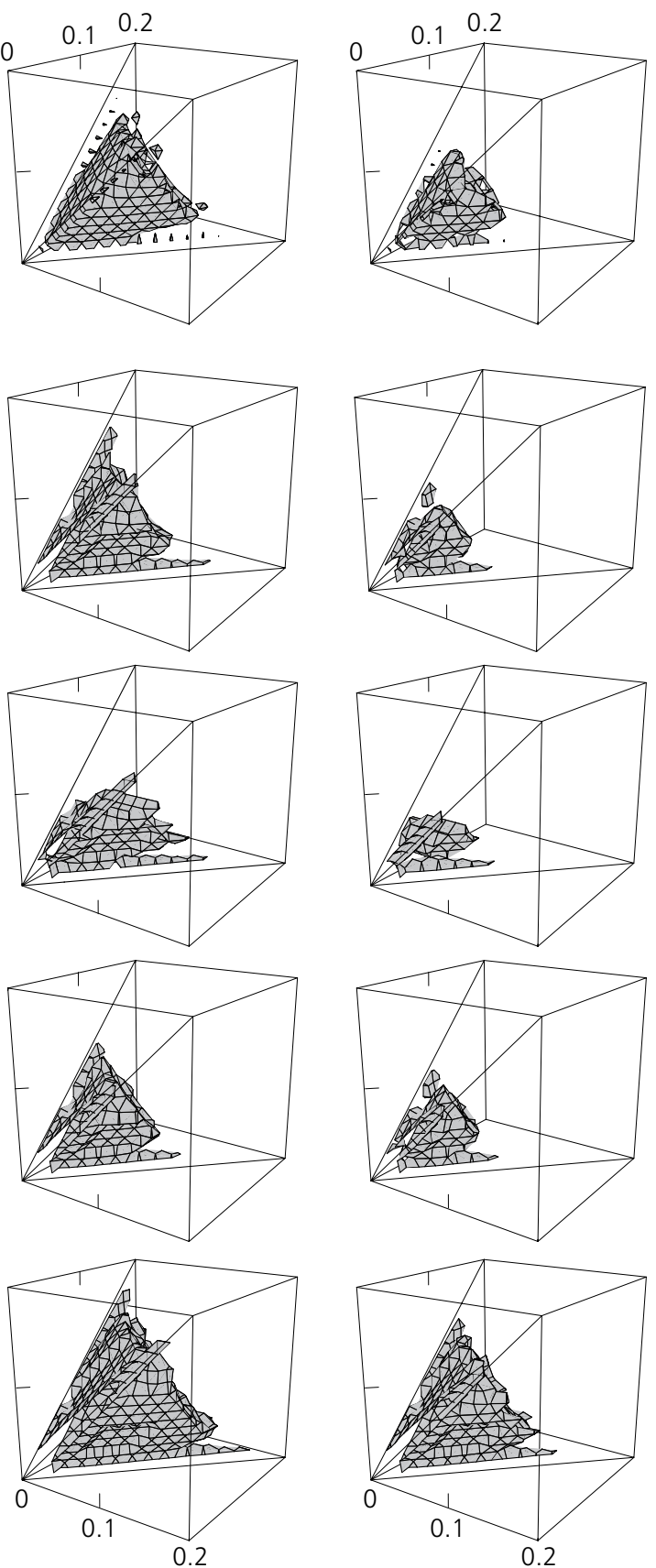

Figure 21.6 Comparison of triplet densities in a single-species system with densities predicted by candidate moment closures. (a) Triplet densities with radial distances $\left(r, r^{\prime}, r^{\prime \prime}\right)$ realized at time $t=100$ in the system described in Figure 21.4 are shown as contour surfaces. Triplet densities can be envisaged as clouds of varying intensity, and contour surfaces of increasing value are nested inside one another; the surfaces shown correspond to triplet densities of 2 (left column), 2.5 (center column), and 3 (right column), all measured relative to a mean-field expectation of 1 . Notice that the three main diagonals of the boundary planes through the origin span a pyramid, outside of which triplet densities are zero - a confinement resulting from the three triangle inequalities for $\left(r, r^{\prime}, r^{\prime \prime}\right)$. Realized singlet and pair densities at time $t=100$ are combined with one of the four moment closures in Equations (21.12a) to (21.12d) and predicted triplet densities are shown in panels (b) to (e), respectively. To diminish the amount of noise, densities are averaged over 100 realizations. 
depicted in Figure 21.6a, based on the same system as in Figure 21.4 at time $t=100$. Figures $21.6 \mathrm{~b}$ to $21.6 \mathrm{e}$ are obtained by applying the moment closures in Equations (21.12a) to (21.12d), respectively, to the mean and pair densities of the individual-based dynamics measured at the same time. All four closures describe the triplet density's shape roughly correctly; it would be difficult to say which one performs best. An obvious discrepancy occurs for the asymmetric power-2 closure, which does not approximate the triplet density for small distances $r^{\prime \prime}$ very well. This is not surprising as, in a triplet, $r^{\prime \prime}$ measures the distance between the two neighbors of a focal individual; the pair correlation density between these neighbors (which can differ from 1 when $r^{\prime \prime}$ is small) does not enter into Equation (21.12b). Ideally, one would want to repeat comparisons as in Figure 21.6 for different points in time, different initial conditions and process parameters, and different numbers of species. Measuring, depicting, and comparing densities in three-dimensional spaces, however, is relatively difficult and time consuming and we therefore turn to describing two alternative types of test.

In a second test, we consider the performance of the candidate closures in predicting time series of mean densities for different parameter settings of a single-species system. When used in conjunction with Equations (21.9) and (21.10), to what extent can the closures forecast the transient and asymptotic behavior exhibited by mean densities? We use three different parameter settings, leading to mean densities that are similar to (Figure 21.7a), lower than (Figure 21.7b), or higher than (Figure 21.7c) those expected from the mean-field approximation. As one would expect, there is little to choose between the closures when the dynamics are close to those of the mean field (Figure 21.7a). Under strong spatial aggregation (Figure $21.7 \mathrm{~b}$ ), the asymmetric power-2 closure performs better than the others. In the case of overdispersion (Figure 21.7c), the symmetric power2 closure performs best.

We extend the comparison of closure performances to predict population dynamics in a two-species system. In a third test, we investigate phase portraits of trajectories for the mean densities of two competing species, with each trajectory starting from a different initial condition. Results of individual-based dynamics are shown in Figure 21.8a. Moment closures in Equations (21.12a) to (21.12d) are used to describe the dynamics of autocorrelation and cross-correlation pair densities, Equation (21.10), which affect the dynamics of mean densities, Equation (21.9). The phase portraits predicted by those moment closures are displayed in Figures 21.8b to 21.8e. In this test, the power- 2 closures clearly perform best and yield accurate 
(a)

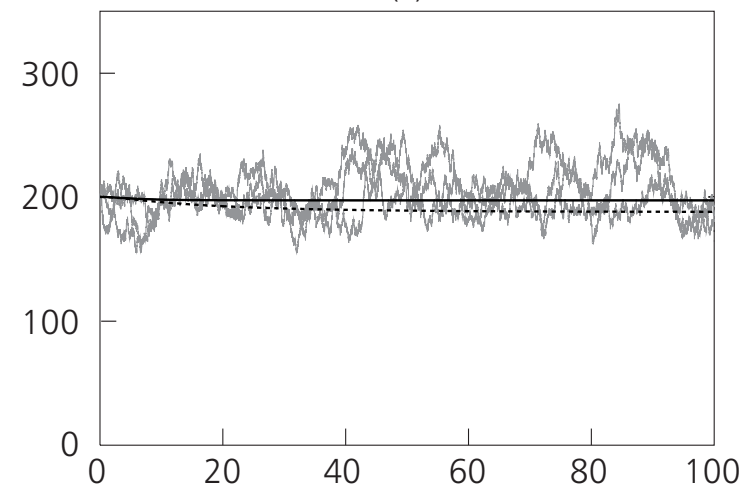

(b)

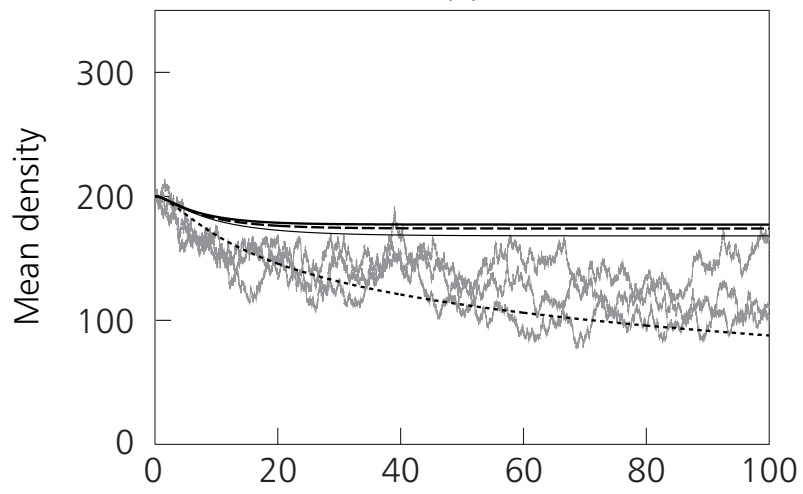

(c)

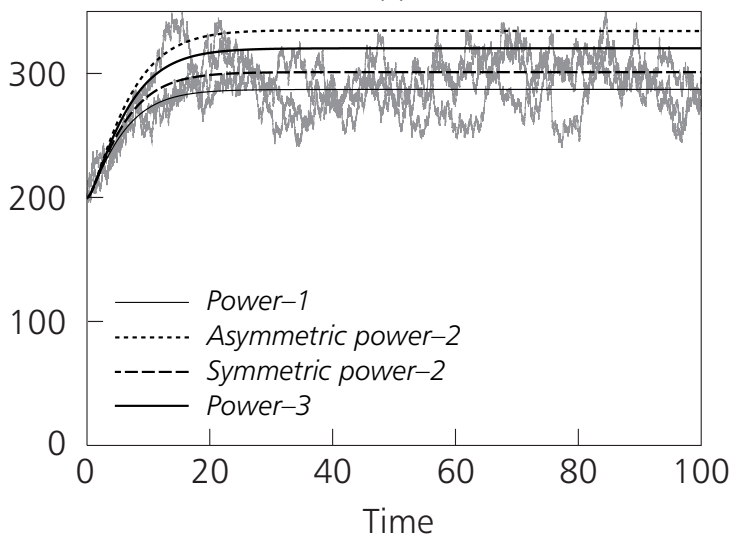

Figure 21.7 Comparison of mean-density dynamics of a single-species system with predictions by candidate moment closures. For a spatial logistic model, three different parameter combinations result in asymptotic mean densities (a) similar to, (b) lower than, or (c) higher than the mean-field equilibrium of $N_{1}=200$. In each case, time series of mean densities for three realizations are shown in gray. Dynamics predicted by pair correlation dynamics in conjunction with one of the moment closures in Equations (21.12a) to $(21.12 \mathrm{~d})$ are superimposed in black. Parameters are the same as in Figure 21.1a, except for $\left(\right.$ a) s.d. $w_{11}^{(d)}=0.15$, s.d. $m_{1}^{(b)}=0.15$; (b) s.d. $w_{11}^{(d)}=0.05$, s.d. $m_{1}^{(b)}=0.05$; (c) s.d. $w_{11}^{(d)}=0.015$, s.d. $m_{1}^{(b)}=0.15$. 
(a)

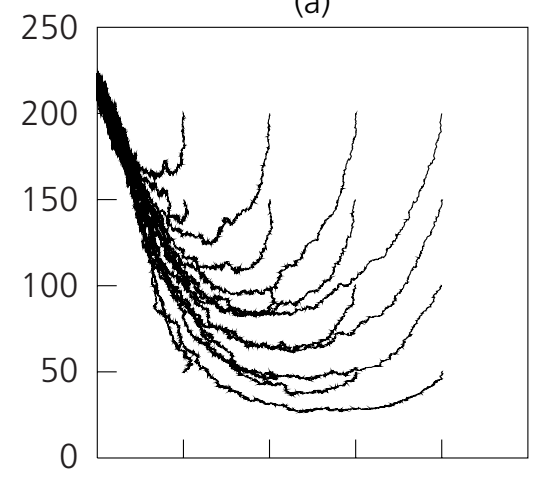

(b)

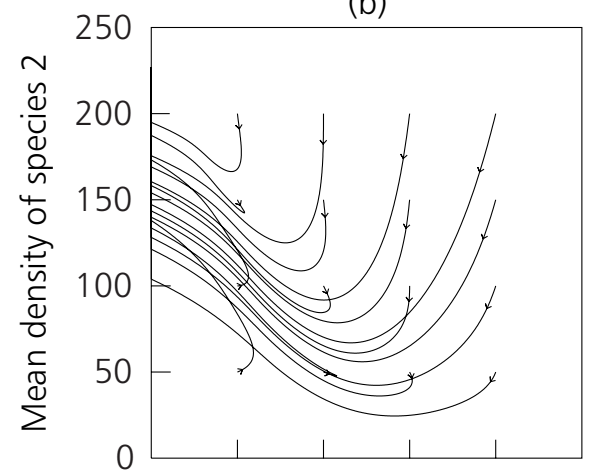

(d)

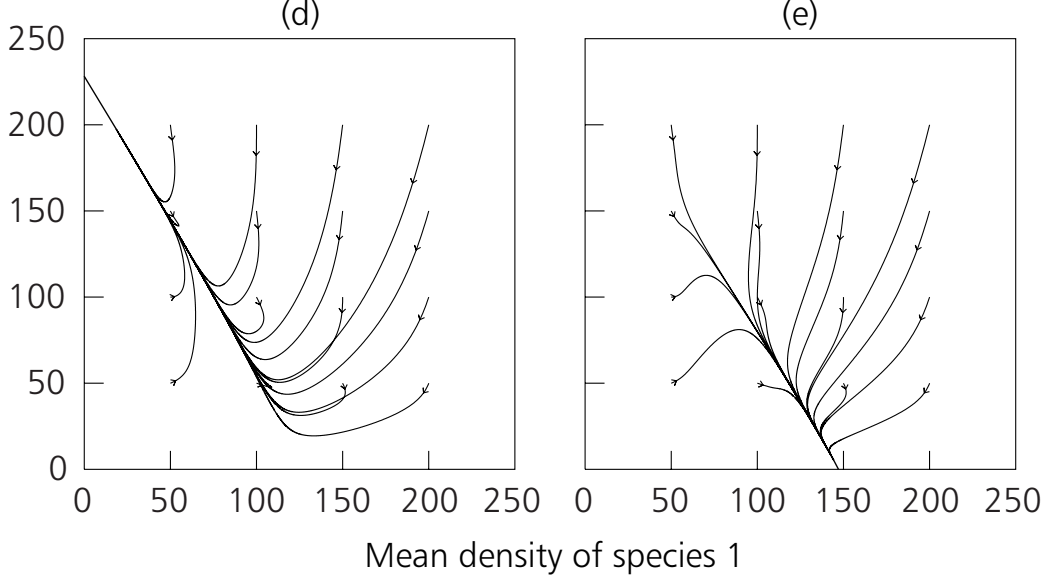

(c)

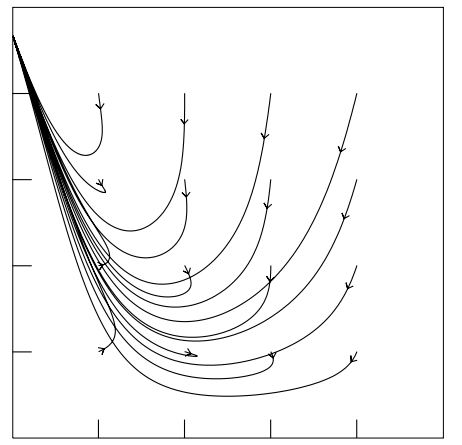

(e)

Figure 21.8 Comparison of phase portraits of a two-species system with predictions by candidate moment closures. Each panel shows trajectories of mean densities $\left(N_{1}, N_{2}\right)$, starting from a grid of 16 initial conditions. (a) Mean paths of the stochastic process, averaged over 20 realizations. (b to e) Phase portraits predicted by pair correlation dynamics in conjunction with one of the moment closures in Equations (21.12a) to (21.12d): (b) power-1 closure; (c) asymmetric power-2 closure; (d) symmetric power2 closure; (e) power-3 closure. Notice that trajectories are projections from higherdimensional dynamics and may thus intersect one another; see also Figure 14.3. Parameters: $b_{1}=b_{2}=0.4, d_{1}=d_{2}=0.2, \underset{(d)}{d_{11}^{\prime}}=d_{22}^{\prime}=0.001, d_{12}^{\prime}=0.005, d_{21}^{\prime}=0.002$, s.d. $w_{11}^{(d)}=$ s.d. $w_{12}^{(d)}=$ s.d. $w_{21}^{(d)}=$ s.d. $w_{22}^{(d)}=$ s.d. $m_{1}^{(b)}=0.03$, s.d. $m_{2}^{(b)}=0.2$. 
portraits of the actual dynamics. In particular, only the asymmetric power2 closure captures the fine details of the phase portrait. Performance of the power- 1 closure is compromised by an instability at small densities, and the power-3 closure even results in prediction of a wrong attractor.

The first test above (summarized in Figure 21.6) is independent of the correlation dynamics and therefore directly probes the accuracy of the moment closures. In contrast, the last two tests (recorded in Figures 21.7 and 21.8) rely on evaluations of the joint performance of closures and correlation dynamics. While a benefit of the first test is its independence of additional theory, more discriminating results and more direct relevance to predictive quality are advantages of the latter pair. On the basis of these tests, we have chosen the asymmetric power- 2 closure for exploring ecological processes in Chapter 14. There is, however, much to be learned about moment closures, and we do not intend to suggest that this particular closure performs best under all circumstances. To gain further insight, there appears to be no alternative to empirical tests for particular ecological settings, in the manner of Figures 21.7 and 21.8.

\subsection{Further Developments and Extensions}

In this chapter we have derived a closed dynamical system for describing spatially heterogeneous change in general ecological communities of interacting species. Instead of considering the simultaneous dynamics of mean and pair densities, Equations (21.9) and (21.10), we can further simplify the description by using Equation (21.10) alone. This is possible because Condition (C2), see Section 21.4, which is satisfied for small-scale spatial patterns, implies $\lim _{|\xi| \rightarrow \infty} \tilde{C}_{i i}(\xi)=N_{i}^{2}$ : consequently, information about mean densities is implicit in the description of pair dynamics. After inserting one of the moment closures from Equations (21.12), Equation (21.10) represents the central result of this chapter and provides a powerful tool for reducing the complexity of individual-based models. We have explained how this result is based on the method of moments: expressing the essential degrees of freedom for a developing spatial pattern in terms of spatial moments and truncating the resulting moment hierarchy using a suitable moment closure yields an approximation for the expected dynamics of moments. The utility of such moment dynamics for describing and understanding ecological processes under spatial heterogeneity is examined in Section 21.4; a variety of successful applications are also presented in Chapter 14. 
To conclude this chapter, we briefly review several directions for further elaborations on the method of moments. These extensions remove some remaining restrictions of the results presented and broaden the scope of ecological systems amenable to simplifying spatial complexity.

\section{External heterogeneity and internal states}

Moment dynamics can account for small-scale spatial heterogeneity in the external environment of populations. As discussed in Section 4.3, which variables are considered external to a system depends on how the system's boundaries are defined. On a short time scale, for example, the concentration of soil nutrients in a spatially extended plant community may be fixed in time and thus can be considered external to the system. In the long run, however, we expect feedback between the dynamics of plants and nutrients. Another example is the effect that landscape patterns have on the dispersal behavior of animals. Some butterflies, for instance, inhabit meadows that arise in gaps created by forest fires, and while the meadows are easily traversed, stretches of forest act as dispersal barriers. In this case, there is no significant feedback between the dynamics of butterflies and forest trees. With or without such feedback, spatial heterogeneity of environmental conditions encountered by a focal population can be formally treated on par with the heterogeneity arising from the presence of extra populations. Straightforward generalizations of Equations (21.10) therefore account for a population's unfolding against the backdrop of a heterogeneous landscape. Salient state variables are the auto-correlations of the focal population, complemented by the auto-correlations of environmental conditions (these may be variable or fixed) as well as the cross-correlations between the population and its environment. Moment dynamics of this kind can provide insights into complex interactions between internal and external mechanisms of ecological pattern formation.

Another extension of moment dynamics allows the incorporation of details about the state of individuals. For example, instead of characterizing a population of trees just by the locations on which they are centered, the size of each tree (height or crown diameter) can be accounted for as well. Implications of asymmetric competition for light, for instance, can thus be studied in spatially heterogeneous settings. Also, a plea for dynamic neighborhoods (see Section 2.5) that vary with, for example, plant size can be met in this way. Other such internal states by which location-based information can be augmented are the age of individuals, their physiological status, or their phenotype. In each case, pair correlation densities take 
two extra arguments, measuring the internal state of paired individuals in addition to their distance. Merging physiologically structured and spatially structured population models in such a way is not easy; yet, such integration would bring models closer to the reality of field systems and is therefore an important path to follow in developing ecological theory.

\section{Fluctuation and correlation corrections}

The importance of taking an "individual's-eye view" in determining the response of a population to its environment has already been emphasized. In this context, we must realize that, for populations of finite density, any bounded neighborhood around an individual will comprise only a finite number of individuals. When numbers of neighbors are large, resulting sampling variation is negligible. But for many systems, neighbors around each individual are few enough for the latter to experience substantially varying local environments. We thus have to ask whether and how such fluctuations feed through to population dynamics and their description by moment equations. The answer comes in three steps.

We observe, first, that local fluctuations do not bear on the expected dynamics of moments if individuals respond linearly to the local densities of individuals in their surroundings. Equations (21.1a) and (21.1c) are based on this assumption - per capita birth and death rates in these spatial analogues of Lotka-Volterra competition depend linearly on the pattern of individuals - and therefore no fluctuation-related terms arise in Equations (21.9) or (21.10). Under such circumstances, the mean response of a population to a distribution of environments equals the response predicted for the distribution mean; consequently, local fluctuations have no effect.

Second, even in the absence of spatial structure (i.e., without pair correlations), the behavior of a well-mixed population of individuals that respond nonlinearly to relatively small numbers of neighbors is different from that of a population under mean-field conditions. A typical result is explained in Box 21.5.

Third, we can investigate the implications of nonlinear responses of individuals to local fluctuations in the presence of pair correlations. Let us consider a single species for which per capita birth and death rates are nonlinearly dependent on local densities, so that, analogous to Equations (21.1a) and (21.1c), we have $D(x, p)=d(n(x, p))$ and $B\left(x, x^{\prime}, p\right)=b(n(x, p)) m^{(b)}\left(x^{\prime}-x\right)$, with local densities $n(x, p)=$ $\int w\left(x^{\prime \prime}-x\right)\left[p\left(x^{\prime \prime}\right)-\delta_{x}\left(x^{\prime \prime}\right)\right] d x^{\prime \prime}$. Under mean-field conditions, the global mean density simply changes according to $\frac{d}{d t} N=f(N) N$, with a per 
Box 21.5 Fluctuation corrections in well-mixed systems

Even in the absence of spatial structure, fluctuations in the local environments of individuals can cause departures from mean-field predictions if individuals respond nonlinearly to their environment.

As an example (R. Ferrière, personal communication; Leitner, unpublished), consider a completely randomly distributed population of individuals, whose recruitment from one season to the next is affected by the number of competing neighbors.

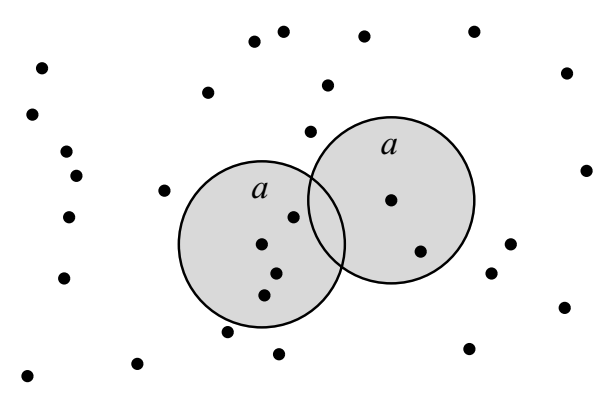

For a mean density $N$ and a uniform weighting of competitors within a neighborhood area $a$, the average number of neighbors equals $a N$. Around this average, neighbor numbers vary according to a Poisson distribution: the probability of finding $n$ neighbors is $P_{n}=\frac{1}{n !}(a N)^{n} e^{-a N}$. If we assume that recruitment in the absence of any competition is $R_{0}$ and that it decreases by a factor $c$ with each competing neighbor, then for $n$ neighbors we have $R_{n}=R_{0} c^{n}$. The expected per capita offspring number is thus given by $\langle R\rangle=\sum_{n=0}^{\infty} R_{n} P_{n}=R_{0} e^{-a N} \sum_{n=0}^{\infty} \frac{1}{n !}(a c N)^{n}=R_{0} e^{-a N} e^{a c N}=$ $e^{\ln R_{0}-a(1-c) N}$. If the between-season dynamics depend entirely on recruitment, the next season's mean density is $N^{\prime}=N\langle R\rangle=N e^{r(1-N / K)}$, with $r=\ln R_{0}$ and $K=\frac{\ln R_{0}}{a(1-c)}$. This is the famous Ricker equation (Ricker 1954) and is quite different from $N^{\prime}=N R_{0} c^{a N}$, the result expected in the absence of fluctuations.

Results of this type are also available for more complicated interactions between individuals (Durrett and Levin 1994b; Czárán 1997) and are always important if individuals respond nonlinearly to small numbers of neighbors. Notice that even though the systems described by these models are assumed to be well mixed, the discreteness of individuals and the finiteness of population densities can result in substantial departures from mean-field predictions. To distinguish such corrections of mean-field results from those arising from spatial structure, we refer to the former as fluctuation corrections and to the latter as correlation corrections. 
capita growth rate $f=b-d$. With local fluctuations and correlations, however, reasoning analogous to that in Section 21.3 shows that the meanfield result ought to be replaced with $\frac{d}{d t} N=\left[f(\bar{n})+\frac{1}{2} \sigma^{2} f^{\prime \prime}(\bar{n})\right] N$, with an average $\bar{n}=N^{-1} \int w(\xi) \tilde{C}(\xi) d \xi$ and a variance $\sigma^{2}$ of local densities. Comparing both dynamics for the global mean density, we observe two corrections relative to the mean-field result. First, a correlation correction replaces the global mean density $N$ with the average local density $\bar{n}$ in the argument of the response function $f$. This reflects the fact that, in the presence of correlations, the average environment around individuals is different from the environment averaged over the entire habitat. Without pair correlations, we have $\tilde{C}(\xi)=N^{2}$ [see Equation (21.11)], which yields $\bar{n}=N$, thus recovering the mean-field result. Second, a fluctuation correction adds the extra term $\frac{1}{2} \sigma^{2} f^{\prime \prime}(\bar{n})$ to the population's response function. Notice that the sign of the term and thus the direction of this correction depends on the response function's curvature at the average local density. We also see that this term vanishes if there are no fluctuations in local densities $\left(\sigma^{2}=0\right)$ or if the response function is linear $\left(f^{\prime \prime}=0\right)$.

\section{Other spatial statistics and further reductions of complexity}

Low-order correlation densities often capture the essential degrees of freedom in spatial ecological processes. For isotropic systems, spatial complexity can be simplified even further by considering angular averages of pair densities, as explained in Section 21.3 and Figure 21.2. Such a transformation of Equation (21.10) then describes pair densities as a function of radial distance instead of vectorial distance. One further step in removing non-essential degrees of freedom can be accomplished by a short-range expansion of Equation (21.10), approximating pair dynamics by the dynamics of intercept, slope, and curvature of pair densities at distance zero. That slope in particular is often a good measure of a pattern's correlation length, characterizing how rapidly pair correlations decay with distance. Correlation lengths can change in the course of ecological dynamics and thus carry key information about patterns as well as processes.

These extensions emphasize that alternative kinds of spatial statistics may be most appropriate for describing a given ecological system. In fact, correlation functions are not the only choice of statistics suitable for simplifying spatial complexity - for particular systems, other relaxation projections may offer superior performance. Chapter 15 provides an example by constructing a patch-based approximation of a spatial host-parasite system. Instead of tracing through time the probabilistic cellular-automaton 
dynamics of that system, only the expected average abundances of species in patches of different types are considered, thus yielding a deterministic description of substantially reduced complexity. Other examples are the socalled local structure approximations of Hiebeler (1997) and the pair-edge approximation of Ellner et al. (1998). The latter approach is especially suited to a class of large-scale spatial patterns where two types of populations live in adjacent domains and a traveling invasion wave develops at their interface (see also Chapters 16, 17, 22, and 23); for such patterns, the position of the invasion front is the central degree of freedom.

This chapter has introduced a general theory of small-scale heterogeneity in ecological processes that is based on correlation densities. Eventually, a tool kit of successful relaxation projections and associated spatial statistics should become available to help ecologists reduce various kinds of complex spatio-temporal models to manageable approximations.

\section{Appendix 21.A Derivation of Pair Dynamics}

In this appendix we explain how to derive the dynamics of corrected pair densities, presented in Equation (21.10).

The expected rates of change in corrected pair densities $\tilde{C}_{i j}(\xi)$ are determined by the first jump moments of these densities, see Equation (21.6c),

$$
\frac{d}{d t} \tilde{C}_{i j}(\xi)=\int a_{i j}(\xi, p) P(p) d p
$$

with contributions coming from birth, death, and movement events,

$$
a_{i j}(\xi, p)=a_{i j}^{(b)}(\xi, p)+a_{i j}^{(d)}(\xi, p)+a_{i j}^{(m)}(\xi, p) .
$$

Let us focus on the birth contribution first. From Equation (21.6c) and Equation (21.2b), we have

$$
\begin{aligned}
a_{i j}^{(b)}(\xi, p) & =\int\left[\tilde{C}_{i j}\left(\xi, p^{\prime}\right)-\tilde{C}_{i j}(\xi, p)\right] \\
& \times \sum_{k} \iint B_{k}\left(x, x^{\prime}, p\right) p_{k}(x) \Delta\left(p+u_{k} \delta_{x^{\prime}}-p^{\prime}\right) d x d x^{\prime} d p^{\prime},
\end{aligned}
$$

which, after collapsing the integration over $p^{\prime}$ using the definition of the generalized delta function (see Box 21.4), simplifies to

$$
\begin{aligned}
a_{i j}^{(b)}(\xi, p) & =\sum_{k} \iint\left[\tilde{C}_{i j}\left(\xi, p+u_{k} \delta_{x^{\prime}}\right)-\tilde{C}_{i j}(\xi, p)\right] \\
& \times B_{k}\left(x, x^{\prime}, p\right) p_{k}(x) d x d x^{\prime} .
\end{aligned}
$$


With $\tilde{C}_{i j}\left(\xi, p+u_{k} \delta_{x^{\prime}}\right)=\frac{1}{A} \int\left(p+u_{k} \delta_{x^{\prime}}\right)_{i}(x)\left[\left(p+u_{k} \delta_{x^{\prime}}\right)_{j}(x+\xi)-\delta_{i j} \delta_{x}(x+\right.$ $\xi)] d x=\frac{1}{A} \int\left[p_{i}(x)+\delta_{i k} \delta_{x^{\prime}}(x)\right]\left[p_{j}(x+\xi)+\delta_{j k} \delta_{x^{\prime}}(x+\xi)-\delta_{i j} \delta(\xi)\right] d x=$ $\tilde{C}_{i j}(\xi, p)+\frac{1}{A}\left[\delta_{j k} p_{i}\left(x^{\prime}-\xi\right)+\delta_{i k} p_{j}\left(x^{\prime}+\xi\right)+\delta_{i k} \delta_{j k} \delta(\xi)-\delta_{i k} \delta_{i j} \delta(\xi)\right]=\tilde{C}_{i j}(\xi, p)+$ $\frac{1}{A}\left[\delta_{j k} p_{i}\left(x^{\prime}-\xi\right)+\delta_{i k} p_{j}\left(x^{\prime}+\xi\right)\right]$, we obtain

$$
\begin{aligned}
a_{i j}^{(b)} & (\xi, p)= \\
& =\sum_{k} \frac{1}{A} \iint\left[\delta_{j k} p_{i}\left(x^{\prime}-\xi\right)+\delta_{i k} p_{j}\left(x^{\prime}+\xi\right)\right] B_{k}\left(x, x^{\prime}, p\right) p_{k}(x) d x d x^{\prime} \\
& =\frac{1}{A} \iint\left[p_{i}\left(x^{\prime}-\xi\right) B_{j}\left(x, x^{\prime}, p\right) p_{j}(x)+p_{j}\left(x^{\prime}+\xi\right) B_{i}\left(x, x^{\prime}, p\right) p_{i}(x)\right] d x d x^{\prime} \\
& =\frac{1}{A} \iint p_{i}(x) p_{j}\left(x^{\prime}+\xi\right) B_{i}\left(x, x^{\prime}, p\right) d x d x^{\prime}+\langle i, j, \xi \rightarrow j, i,-\xi\rangle
\end{aligned}
$$

As in Equation (21.10), the expression $\langle i, j, \xi \rightarrow j, i,-\xi\rangle$ is shorthand and stands for all its preceding terms after changing $i$ to $j, j$ to $i$, and $\xi$ to $-\xi$. Inserting $B_{i}$ according to Equation (21.1c) yields

$$
\begin{aligned}
a_{i j}^{(b)}(\xi, p) & =\frac{1}{A} \iint p_{i}(x) p_{j}\left(x^{\prime}+\xi\right)\left[b_{i}+\sum_{k} b_{i k}^{\prime} \int w_{i k}^{(b)}\left(x^{\prime \prime}-x\right)\right. \\
& \left.\times\left[p_{k}\left(x^{\prime \prime}\right)-\delta_{i k} \delta_{x}\left(x^{\prime \prime}\right)\right] d x^{\prime \prime}\right] m_{i}^{(b)}\left(x^{\prime}-x\right) d x d x^{\prime}+\langle i, j, \xi \rightarrow j, i,-\xi\rangle,
\end{aligned}
$$

or, when separating terms for density-independent and density-dependent birth,

$$
\begin{aligned}
a_{i j}^{(b)}(\xi, p)= & +b_{i} \frac{1}{A} \iint p_{i}(x) p_{j}\left(x^{\prime}+\xi\right) m_{i}^{(b)}\left(x^{\prime}-x\right) d x d x^{\prime} \\
& +\sum_{k} b_{i k}^{\prime} \frac{1}{A} \iiint p_{i}(x) p_{j}\left(x^{\prime}+\xi\right) \\
& \times\left[p_{k}\left(x^{\prime \prime}\right)-\delta_{i k} \delta_{x}\left(x^{\prime \prime}\right)\right] m_{i}^{(b)}\left(x^{\prime}-x\right) w_{i k}^{(b)}\left(x^{\prime \prime}-x\right) d x d x^{\prime} d x^{\prime \prime} \\
& +\langle i, j, \xi \rightarrow j, i,-\xi\rangle .
\end{aligned}
$$

The $x$-integration of $p_{i} p_{j}$ in the first term and of $p_{i} p_{j} p_{k}$ in the second resembles the definitions of $\tilde{C}_{i j}$ and $\tilde{T}_{i j k}$ in Equation (21.4a) and (21.4b), respectively. For full compatibility with integrands in these definitions, we must subtract (and then add in again) one self-pair term for $\tilde{C}_{i j}, p_{i}(x) p_{j}\left(x^{\prime}+\xi\right)=p_{i}(x)\left[p_{j}\left(x^{\prime}+\xi\right)-\right.$ $\left.\delta_{i j} \delta_{x}\left(x^{\prime}+\xi\right)\right]+p_{i}(x) \delta_{i j} \delta_{x}\left(x^{\prime}+\xi\right)$, and two such terms for $\tilde{T}_{i j k}, p_{i}(x) p_{j}\left(x^{\prime}+\right.$ $\xi)\left[p_{k}\left(x^{\prime \prime}\right)-\delta_{i k} \delta_{x}\left(x^{\prime \prime}\right)\right]=p_{i}(x)\left[p_{j}\left(x^{\prime}+\xi\right)-\delta_{i j} \delta_{x}\left(x^{\prime}+\xi\right)\right]\left[p_{k}\left(x^{\prime \prime}\right)-\delta_{i k} \delta_{x}\left(x^{\prime \prime}\right)-\right.$ $\left.\delta_{j k} \delta_{x^{\prime}+\xi}\left(x^{\prime \prime}\right)\right]+p_{i}(x) \delta_{i j} \delta_{x}\left(x^{\prime}+\xi\right)\left[p_{k}\left(x^{\prime \prime}\right)-\delta_{j k} \delta_{x^{\prime}+\xi}\left(x^{\prime \prime}\right)\right]+p_{i}(x)\left[p_{j}\left(x^{\prime}+\xi\right)-\right.$ $\left.\delta_{i j} \delta_{x}\left(x^{\prime}+\xi\right)\right] \delta_{j k} \delta_{x^{\prime}+\xi}\left(x^{\prime \prime}\right)$. With $\xi^{\prime}=x^{\prime}-x$ and $\xi^{\prime \prime}=x^{\prime \prime}-x$, we thus obtain 


$$
\begin{aligned}
a_{i j}^{(b)}(\xi, p)= & +b_{i} \int m_{i}^{(b)}\left(\xi^{\prime}\right) \tilde{C}_{i j}\left(\xi+\xi^{\prime}, p\right) d \xi^{\prime} \\
& +\delta_{i j} b_{i} m_{i}^{(b)}(-\xi) N_{i}(p) \\
& +\sum_{k} b_{i k}^{\prime} \iint m_{i}^{(b)}\left(\xi^{\prime}\right) w_{i k}^{(b)}\left(\xi^{\prime \prime}\right) \tilde{T}_{i j k}\left(\xi+\xi^{\prime}, \xi^{\prime \prime}, p\right) d \xi^{\prime} d \xi^{\prime \prime} \\
& +\delta_{i j} m_{i}^{(b)}(-\xi) \sum_{k} b_{i k}^{\prime} \int w_{i k}^{(b)}\left(\xi^{\prime \prime}\right) \tilde{C}_{i k}\left(\xi^{\prime \prime}, p\right) d \xi^{\prime \prime} \\
& +b_{i j}^{\prime} \int m_{i}^{(b)}\left(\xi^{\prime}\right) w_{i j}^{(b)}\left(\xi+\xi^{\prime}\right) \tilde{C}_{i j}\left(\xi+\xi^{\prime}, p\right) d \xi^{\prime} \\
& +\langle i, j, \xi \rightarrow j, i,-\xi\rangle
\end{aligned}
$$

We now turn to calculating the death contribution,

$a_{i j}^{(d)}(\xi, p)=\sum_{k} \int\left[\tilde{C}_{i j}\left(\xi, p-u_{k} \delta_{x}\right)-\tilde{C}_{i j}(\xi, p)\right] D_{k}(x, p) p_{k}(x) d x$.

With $\tilde{C}_{i j}\left(\xi, p-u_{k} \delta_{x}\right)=\frac{1}{A} \int\left[p_{i}\left(x^{\prime}\right)-\delta_{i k} \delta_{x}\left(x^{\prime}\right)\right]\left[p_{j}\left(x^{\prime}+\xi\right)-\delta_{j k} \delta_{x}\left(x^{\prime}+\xi\right)-\right.$ $\left.\delta_{i j} \delta(\xi)\right] d x^{\prime}=\tilde{C}_{i j}(\xi, p)+\frac{1}{A}\left[-\delta_{j k} p_{i}(x-\xi)-\delta_{i k} p_{j}(x+\xi)+\delta_{i k} \delta_{j k} \delta(\xi)+\right.$ $\left.\delta_{i k} \delta_{i j} \delta(\xi)\right]=\tilde{C}_{i j}(\xi, p)-\frac{1}{A}\left\{\delta_{i k}\left[p_{j}(x+\xi)-\delta_{j k} \delta(\xi)\right]+\delta_{j k}\left[p_{i}(x-\xi)-\delta_{i k} \delta(\xi)\right]\right\}$, we obtain

$$
\begin{aligned}
a_{i j}^{(d)}(\xi, p)= & -\frac{1}{A} \int p_{i}(x)\left[p_{j}(x+\xi)-\delta_{i j} \delta(\xi)\right] D_{i}(x, p) d x \\
& +\langle i, j, \xi \rightarrow j, i,-\xi\rangle .
\end{aligned}
$$

After inserting $D_{i}$ according to Equation (21.1a), this yields

$$
\begin{aligned}
a_{i j}^{(d)}(\xi, p)= & -d_{i} \frac{1}{A} \int p_{i}(x)\left[p_{j}(x+\xi)-\delta_{i j} \delta(\xi)\right] d x \\
& -\sum_{k} d_{i k}^{\prime} \frac{1}{A} \iint p_{i}(x)\left[p_{j}(x+\xi)-\delta_{i j} \delta(\xi)\right] \\
& \times\left[p_{k}\left(x^{\prime \prime}\right)-\delta_{i k} \delta_{x}\left(x^{\prime \prime}\right)\right] w_{i k}^{(d)}\left(x^{\prime \prime}-x\right) d x d x^{\prime \prime} \\
& +\langle i, j, \xi \rightarrow j, i,-\xi\rangle .
\end{aligned}
$$

Notice that the integral in the first term matches the definition of $\tilde{C}_{i j}$. To achieve full compatibility with the definition of $\tilde{T}_{i j k}$ in the second term, we subtract (and then add in again) a self-pair term, as we did in calculating the birth contribution: $p_{i}(x)\left[p_{j}(x+\xi)-\delta_{i j} \delta(\xi)\right]\left[p_{k}\left(x^{\prime \prime}\right)-\delta_{i k} \delta_{x}\left(x^{\prime \prime}\right)\right]=p_{i}(x)\left[p_{j}(x+\xi)-\right.$ $\left.\delta_{i j} \delta_{x}(x+\xi)\right]\left[p_{k}\left(x^{\prime \prime}\right)-\delta_{i k} \delta_{x}\left(x^{\prime \prime}\right)-\delta_{j k} \delta_{x+\xi}\left(x^{\prime \prime}\right)\right]+p_{i}(x)\left[p_{j}(x+\xi)-\delta_{i j} \delta_{x}(x+\right.$ $\xi)] \delta_{j k} \delta_{x+\xi}\left(x^{\prime \prime}\right)$. With $\xi^{\prime \prime}=x^{\prime \prime}-x$, we arrive at

$$
\begin{aligned}
a_{i j}^{(d)}(\xi, p)= & -d_{i} \tilde{C}_{i j}(\xi, p) \\
& -\sum_{k} d_{i k}^{\prime} \int w_{i k}^{(d)}\left(\xi^{\prime \prime}\right) \tilde{T}_{i j k}\left(\xi, \xi^{\prime \prime}, p\right) d \xi^{\prime \prime} \\
& -d_{i j}^{\prime} w_{i j}^{(d)}(\xi) \tilde{C}_{i j}(\xi, p) \\
& +\langle i, j, \xi \rightarrow j, i,-\xi\rangle .
\end{aligned}
$$


Finally, we calculate the contribution of movement events to the dynamics of corrected pair densities,

$$
\begin{aligned}
a_{i j}^{(m)}(\xi, p) & =\sum_{k} \iint\left[\tilde{C}_{i j}\left(\xi, p-u_{k} \delta_{x}+u_{k} \delta_{x^{\prime}}\right)-\tilde{C}_{i j}(\xi, p)\right] \\
& \times M_{k}\left(x, x^{\prime}, p\right) p_{k}(x) d x d x^{\prime} .
\end{aligned}
$$

With $\tilde{C}_{i j}\left(\xi, p-u_{k} \delta_{x}+u_{k} \delta_{x^{\prime}}\right)=\frac{1}{A} \int\left[p_{i}\left(x^{\prime \prime}\right)-\delta_{i k} \delta_{x}\left(x^{\prime \prime}\right)+\delta_{i k} \delta_{x^{\prime}}\left(x^{\prime \prime}\right)\right]\left[p_{j}\left(x^{\prime \prime}+\right.\right.$ $\left.\xi)-\delta_{j k} \delta_{x}\left(x^{\prime \prime}+\xi\right)+\delta_{j k} \delta_{x^{\prime}}\left(x^{\prime \prime}+\xi\right)-\delta_{i j} \delta(\xi)\right] d x^{\prime \prime}=\tilde{C}_{i j}(\xi, p)+\frac{1}{A}\left[-\delta_{j k} p_{i}(x-\xi)+\right.$ $\delta_{j k} p_{i}\left(x^{\prime}-\xi\right)-\delta_{i k} p_{j}(x+\xi)+\delta_{i k} \delta_{j k} \delta(\xi)-\delta_{i k} \delta_{j k} \delta_{x^{\prime}}(x+\xi)+\delta_{i k} \delta_{i j} \delta(\xi)+\delta_{i k} p_{j}\left(x^{\prime}+\right.$ $\left.\xi)-\delta_{i k} \delta_{j k} \delta_{x}\left(x^{\prime}+\xi\right)+\delta_{i k} \delta_{j k} \delta(\xi)-\delta_{i k} \delta_{i j} \delta(\xi)\right]=\tilde{C}_{i j}(\xi, p)-\frac{1}{A}\left\{\delta_{i k}\left[p_{j}\left(x^{\prime}+\xi\right)-\right.\right.$ $\left.p_{j}(x+\xi)-\delta_{j k} \delta_{x}\left(x^{\prime}+\xi\right)+\delta_{j k} \delta(\xi)\right]+\delta_{j k}\left[p_{i}\left(x^{\prime}-\xi\right)-p_{i}(x-\xi)-\delta_{i k} \delta_{x}\left(x^{\prime}-\right.\right.$ $\left.\left.\xi)+\delta_{i k} \delta(\xi)\right]\right\}$, and with $M_{i}\left(x, x^{\prime}, p\right)=m_{i}\left(x^{\prime}-x\right)$ from Equation (21.1b), we get

$$
\begin{aligned}
a_{i j}^{(m)}(\xi, p)=+ & \frac{1}{A} \iint p_{i}(x)\left[p_{j}\left(x^{\prime}+\xi\right)-p_{j}(x+\xi)\right. \\
& \left.-\delta_{i j} \delta_{x}\left(x^{\prime}+\xi\right)+\delta_{i j} \delta(\xi)\right] m_{i}\left(x^{\prime}-x\right) d x d x^{\prime} \\
& +\langle i, j, \xi \rightarrow j, i,-\xi\rangle .
\end{aligned}
$$

For matching the definition of $\tilde{C}_{i j}$, we rewrite this equation by splitting the integral in two,

$$
\begin{aligned}
a_{i j}^{(m)}(\xi, p)= & +\frac{1}{A} \iint p_{i}(x)\left[p_{j}\left(x^{\prime}+\xi\right)-\delta_{i j} \delta_{x}\left(x^{\prime}+\xi\right)\right] m_{i}\left(x^{\prime}-x\right) d x d x^{\prime} \\
& -\frac{1}{A} \iint p_{i}(x)\left[p_{j}(x+\xi)-\delta_{i j} \delta(\xi)\right] m_{i}\left(x^{\prime}-x\right) d x d x^{\prime} \\
& +\langle i, j, \xi \rightarrow j, i,-\xi\rangle .
\end{aligned}
$$

With $\xi^{\prime}=x^{\prime}-x$, this yields

$$
\begin{aligned}
a_{i j}^{(m)}(\xi, p)= & +\int m_{i}\left(\xi^{\prime}\right) \tilde{C}_{i j}\left(\xi+\xi^{\prime}, p\right) d \xi^{\prime} \\
& -\tilde{C}_{i j}(\xi, p) \int m_{i}\left(\xi^{\prime}\right) d \xi^{\prime} \\
& +\langle i, j, \xi \rightarrow j, i,-\xi\rangle .
\end{aligned}
$$

Inserting the three results from Equations (21.14f), (21.15d), and (21.16d) into Equations (21.13) recovers the dynamics of corrected pair densities, Equation (21.10). 


\section{References}

References in the book in which this chapter is published are integrated in a single list, which appears on pp. 517-552. For the purpose of this reprint, references cited in the chapter have been assembled below.

Anderson RM \& May RM (1991). Infectious Diseases of Humans: Dynamics and Control. Oxford, UK: Oxford University Press

Bailey NTJ (1975). The Mathematical Theory of Infectious Diseases and Its Applications. London, UK: Griffin

Bolker B \& Pacala SW (1997). Using moment equations to understand stochastically driven spatial pattern formation in ecological systems. Theoretical Population Biology 52:179-197

Dieckmann U (1994). Coevolutionary Dynamics of Stochastic Replicator Systems. Juelich, Germany: Central Library of the Research Center Juelich

Dieckmann U, Herben T \& Law R (1997). Spatio-temporal processes in plant communities. In Yearbook 1995/96, Institute for Advanced Study Berlin, ed. Lepenies W, pp. 296-326. Berlin, Germany: Nicolaische Verlagsbuchhandlung

Dieckmann U, Herben T \& Law R. Spatio-temporal processes in ecological communities. CWI Quarterly. In press

Diggle PJ (1983). Statistical Analysis of Spatial Point Patterns. London, UK: Academic Press

Dirac PAM (1926). The physical interpretation of the quantum dynamics. Proceedings of the Royal Society of London A 113:621-641

Dirac PAM (1958). The Principles of Quantum Dynamics, 4th edn. Oxford, UK: Clarendon Press

Ellner SP, Sasaki A, Haraguchi Y \& Matsuda H (1998). Speed of invasion in lattice population models: Pair-edge approximation. Journal of Mathematical Biology 36(5):469-484

Hiebeler D (1997). Stochastic spatial models: From simulations to mean field and local structure approximations. Journal of Theoretical Biology 187:307-319

Hogeweg P (1988). Cellular automata as a paradigm for ecological modeling. Applied Mathematics and Computation 27:81-100

Holsinger KE \& Roughgarden J (1985). A model for the dynamics of an annual plant-population. Theoretical Population Biology 28:288-313

Jammer M (1966). The Conceptual Development of Quantum Mechanics. New York, NY, USA: McGraw-Hill

Kirkwood JG (1935). Statistical mechanics of fluid mixtures. Journal of Chemical Physics 3:300-313

Leitner W. From individual life history processes to population dynamics: a derivation of the Ricker map. Unpublished

Levin SA (1974). Dispersion and population interactions. The American Naturalist 108:207-228

Levin SA (1976). Population dynamical models in heterogeneous environments. Annual Review of Ecology and Systematics, Vol. 7, pp. 287-310 
Lewontin RC (1974). The Genetic Basis of Evolutionary Change. New York, NY, USA: Columbia University Press

Mahdi A \& Law R (1987). On the spatial organization of plant species in a limestone grassland community. Journal of Ecology 75:459-476

Pacala SW (1986). Neighborhood models of plant population dynamics. II. Multispecies models of annuals. Theoretical Population Biology 29:262-292

Pacala SW \& Silander JA Jr (1985). Neighborhood models of plant population dynamics. I. Single-species models of annuals. The American Naturalist 125:385-411

Ricker WE (1954). Stock and recruitment. Journal of the Fisheries Research Board of Canada 11: 559-623

Ripley BD (1981). Spatial Statistics. New York, NY, USA: Wiley

Schwartz L (1950). Théorie des distributions. Paris, France: Hermann

Turkington RA \& Harper JL (1979). The growth, distribution and neighbour relationships of Trifolium repens in a permanent pasture. I. Ordination, pattern and contact. Journal of Ecology 67:201-218

Turkington RA \& Harper JL (1979). The growth, distribution and neighbour relationships of Trifolium repens in a permanent pasture. I. Ordination, pattern and contact. Journal of Ecology 67:201-218

van Kampen NG (1992). Stochastic Processes in Physics and Chemistry. Amsterdam, Netherlands: North-Holland

Watt AS (1947). Pattern and process in the plant community. Journal of Ecology 35:1-22

Weinberger HF (1982). Long-time behaviour of a class of biological models. SIAM Journal of Mathematical Analysis 13:353-396

Weiner J (1982). A neighborhood model of annual-plant interference. Ecology 63: $1237-1241$

Weiner J \& Conte PT (1981). Dispersal and neighborhood effects in an annual plant competition model. Ecological Modelling 13:131-147

Ziman JM (1979). Models of Disorder: The Theoretical Physics of Homogeneously Disordered Systems. Cambridge, UK: Cambridge University Press 